\title{
WEAK* SOLUTIONS I: A NEW PERSPECTIVE ON SOLUTIONS TO SYSTEMS OF CONSERVATION LAWS
}

\author{
Alexey Miroshnikov \& Robin Young
}

\begin{abstract}
We introduce a new notion of solution, which we call weak* solutions, for systems of conservation laws. These solutions can be used to handle singular situations that standard weak solutions cannot, such as vacuums in Lagrangian gas dynamics or cavities in elasticity. Our framework allows us to treat the systems as ODEs in Banach space. Starting with the observation that solutions act linearly on test functions $\alpha \in X$, we require solutions to take values in the dual space $X^{*}$ of $X$. Moreover, we weaken the usual requirement of measurability of solutions. In order to do this, we develop the calculus of the Gelfand integral, which is appropriate for weak* measurable functions. We then use the Gelfand calculus to define weak* solutions, and show that they are stronger than the usual notion of weak solution, although for $B V$ solutions the notions are equivalent. It is expected that these solutions will also shed light on vexing issues of ill-posedness for multi-dimensional systems.
\end{abstract}

\section{Introduction}

Hyperbolic systems of conservation laws are fundamental in the study of fluids and continuum dynamics, expressing basic physical properties such as conservation of mass and momentum. In one dimension, these take the form

$$
u_{t}+F(u)_{x}=0, \quad u(x, 0)=u^{0}(x),
$$

where $u \in \mathcal{U} \subset \mathbb{R}^{n}, F: \mathcal{U} \rightarrow \mathbb{R}^{n}$, being derived from the integral form

$$
\frac{d}{d t} \int_{(a, b)} u d x=-\int_{\partial(a, b)} F \cdot n d s=-\left.F(u)\right|_{a} ^{b} .
$$

Here $u$ is the vector of conserved quantities, and $F$ is the corresponding flux. Mathematically, these equations present particular challenges because of the ubiquity of shock waves, at which continuum fields such as pressure are discontinuous, so that global classical solutions generally do not exist.

The study of weak solutions for general systems was initiated by Peter Lax in the 1950's 11, and followed in the 1960's by Glimm's celebrated proof of the global existence of weak solutions, provided the initial data has sufficiently small total variation [9]. A weak solution is a locally bounded, locally

Mathematics Subject Classification. 35L65, 35L67, 46G12, 28B05, 35L40, 35L90.

Key words and phrases. conservation laws, shock waves, Gelfand integral. 
integrable function $u$ satisfying

$$
\begin{aligned}
\int_{0}^{T} \int_{\mathbb{R}}\left(u(x, t)^{\top} \partial_{t} \varphi(x, t)\right. & \left.+F(u(x, t))^{\top} \partial_{x} \varphi(x, t)\right) d x d t \\
& +\int_{\mathbb{R}} u^{0}(x)^{\top} \varphi(0, x) d x=0,
\end{aligned}
$$

for all smooth test functions $\varphi$ [1, 5. This must be augmented by an entropy condition, which serves to restrict weak solutions to those which contain only admissible shocks. There is now a mature and complete theory of entropy weak solutions in one space dimension, culminating in Bressan and collaborators' proofs of uniqueness and continuous dependence of entropy weak solutions, both via approximation schemes and via vanishing viscosity [2, 1. All of these results depend on Glimm's global $B V$ estimates, which in turn require the initial data to have small amplitude and bounded variation $\mathbf{2 3}$.

If the data is large, even for physical systems, the class of weak solutions is not always sufficient to cope with the variety of solutions. In particular, the use of a Lagrangian frame, which is often computationally convenient, presents problems near vacuums, cavities and fractures in the medium, where they are represented by Dirac masses [24, 25]. There have been several varied attempts to extend the class of weak solutions in these and similar contexts [10, 6, 20, 8. This problem is worse in higher dimensions, for which there are several instances of solutions exhibiting instability and nonuniqueness, independent of entropy considerations $[\mathbf{1 7}, \mathbf{1 9}, \mathbf{2 1}, \mathbf{1 2}$.

Our initial motivation was to allow Dirac masses, representing vacuums, which are discontinuities in the medium, to appear in solutions of the Euler equations of gas dynamics, in a Lagrangian frame. In particular, once the use of Dirac masses is rigorously justified, their use simplifies the manipulation of solutions in calculations. In addition, we would like to be able to rigorously describe solutions as the solutions of an ODE in a Banach space, thus correctly regarding the conservation law as an evolution equation. We would also prefer a definition that more closely resembles the integral form of the balance law than does (2).

The pairing (2) of the weak solution $u(x, t)$ with arbitrary smooth test functions $\varphi \in C_{c}^{\infty}(\mathbb{R} \times(0, T))$, together with the idea of studying the evolution $t \rightarrow u(t)=u(\cdot, t)$, motivate the following observations.

- $u(t)$, the value of a solution $u$, should be a spatial function which acts linearly on spatial test functions $x \rightarrow \alpha(x)$, that live in a certain Banach space $X$. Thus, $u(t)$ should take values in the dual space, $u(t) \in X^{*}$.

- Rather than requiring $t \rightarrow u(t) \in X^{*}$ to be (strongly) Bochner integrable, it is sufficient to require the weaker condition that the scalar function $t \rightarrow\langle u(t), \alpha\rangle$ be integrable for each test function $\alpha \in X$.

- To make sense of the nonlinear flux $F(u)$ and its derivative in the space $X^{*}$, one needs to properly define the flux as a mapping $F: X^{*} \rightarrow X^{*}$, so $F$ must be extended to a map of Banach spaces.

- To obtain consistency and be able to use the calculus of distributions, we also should require that a union of sets $C_{c}^{\infty}(\Omega)$ are dense in $X$ for appropriate subsets $\Omega \subseteq \mathbb{R}$. 
In this paper, we develop the point of view that $u=u(t) \in X^{*}$ is a map on $[0, T]$ with values in a Banach space $X^{*}$, which solves the evolution equation

$$
u^{\prime}(t)+\mathbb{D}_{x} F(u(t))=0 \text { in } X^{*},
$$

in a natural sense, for appropriately interpreted time derivative $u^{\prime}$ and flux $F(u)$. That is, we view the problem as a time evolution, treating space using the relation between the test function space $X$ and its dual $X^{*}$, while for time we use the usual test functions $C_{c}^{\infty}(0, T)$. We note that weak solutions, defined by (2), make no distinction between time and space.

In light of our second observation above, we regard the assumption of strong measurability, in which $u:[0, T] \rightarrow X^{*}$ is measurable, as being too strong for our purposes. Instead, we prefer to use the concept of weak* measurability, so that the scalar function $\langle u, \alpha\rangle:[0, t] \rightarrow \mathbb{R}$ is measurable for each $\alpha \in X$. The appropriate notion of integration in this case is the Gelfand integral, denoted $\star \int_{E} u(t) d t$, with $E \in \mathfrak{B}([0, T])$ measurable, which is essentially defined by the condition

$$
\star \int_{E} u(t) d t \in X^{*}, \quad \text { such that }\left\langle\star \int_{E} u(t) d t, \alpha\right\rangle=\int_{E}\langle u(t), \alpha\rangle d t,
$$

for all spatial test functions $\alpha \in X$. In fact, in order to make sense of the solution $u(t)$ as a measure, we are required to use the Gelfand integral rather than the Bochner integral.

Although the Gelfand integral goes back to the 1930's, to the authors' knowledge it has not been extensively studied. In this paper we recall the Gelfand integral and develop the calculus thereof, including appropriate versions of the Fundamental Theorem of Calculus and an integration by parts formula. We then develop a related notion of Gelfand weak (G-weak) derivative, and introduce the space $W_{w *}^{1, q}\left(0, T ; X^{*}\right)$ as the analogue of the usual space $W^{1, q}$ of weakly differentiable functions; in particular, our analysis shows that functions from $W_{w *}^{1, q}\left(0, T ; X^{*}\right)$ have absolutely continuous representatives with weak derivatives in the space of weak* measurable maps $L_{w *}^{q}\left(0, T ; X^{*}\right)$, the dual of $L^{q^{\prime}}(0, T ; X)$.

Having developed the calculus of the Gelfand integral, we define the notion of weak ${ }^{*}$ solution. Here weak* refers to the target space rather than any type of convergence. Suppose that the initial data for (1) is $u^{0} \in X^{*}$. We say that the function $u \in W_{w *}^{1, q}\left(0, T ; X^{*}\right)$ is a weak ${ }^{*}$ solution to (1), if

$$
\bar{u}(t)-\bar{u}(s)=\star \int_{s}^{t} \mathbb{D}_{x} F(u(\tau)) d \tau, \quad \text { in } \quad X^{*},
$$

for $t, s \in[0, T]$, and $\bar{u}(0)=u^{0}$ in $X^{*}$. Here $\bar{u}$ is the absolutely continuous representative of $u$ with values in $X^{*}$, and we must make sense of the flux map $F: X^{*} \rightarrow X^{*}$ and distributional derivative operator $\mathbb{D}_{x}$, while implicitly requiring $\mathbb{D}_{x} F(u(\cdot)) \in X^{*}$. The formulation (4) is equivalent to that of (3), interpreting $u^{\prime}$ as the $G$-weak derivative of $u$. Moreover, (4) provides a more direct interpretation of the system (11) than (2), essentially because the integration by parts is carried out implicitly and abstractly in the definition of the spaces $W_{w *}^{1, q}\left(0, T ; X^{*}\right)$.

We note that, unlike the usual definition of weak solution, our definition implicitly imposes a certain regularity on the solution $u$, in that $t \rightarrow u(t)$ must 
be absolutely continuous in $X^{*}$. Also, although we require $\mathbb{D}_{x} F(u(t)) \in X^{*}$, this need only be weak* measurable, rather than strongly measurable as a function of $t$. More precisely, the mapping

$$
t \rightarrow \mathbb{D}_{x} F(u(t)) \quad \text { belongs to } L_{w *}^{q}\left(0, T ; X^{*}\right)=L^{q^{\prime}}(0, T ; X)^{*} .
$$

Note also that we allow any $1 \leq q \leq \infty$, so we allow different rates of growth of solutions. When weak* solutions contain discontinuties such as shocks, we are again required to rule out inadmissible shocks via entropy considerations; when an entropy-flux pair is available, the entropy inequality is again interpreted in the weak* sense.

In principle, by changing the space $X$ of spatial test functions, we could admit different classes of weak* solutions. As an example, classical $H^{s}$ solutions are obtained by setting $X=H^{-s}$, a large space, while by restricting the class of test functions to a smaller space, say $X=H^{s}$, we allow more solutions. In [14, the authors choose $X=C_{0}$, so that weak* solutions can include Radon measures, which allows the treatment of vacuums in gas dynamics and fractures in elasticity. These weak* solutions are not weak solutions as they include Dirac masses; on the other hand, the implicit regularity of weak* solutions means that weak* solutions are more restrictive than weak solutions.

In the present work, we primarily focus on weak* solutions $u \in X^{*} \cap B V^{n}$, with $X=\left(C_{0}\right)^{n}$ and $X^{*}=M^{n}$, which we call $B V$ weak* solutions. Having defined this class of solutions, we compare them to the more familiar entropy weak solutions. We prove that weak* solutions are distributional solutions, essentially because the product test functions $\alpha(x) \beta(t)$ are dense in the set $C_{c}^{\infty}\left(\mathbb{R}^{2}\right)$. In particular, a locally bounded weak* solution is a weak solution.

Next, we prove that a weak solution with appropriate a.e. bounds on the spatial total variation is a weak* solution, and in particular, any global solution obtained via Glimm's method or vanishing viscosity is a global weak* solution with $q=\infty$. This means that the celebrated well-posedness theory for small $B V$ weak solutions developed by Bressan's school applies without change to weak* solutions. Indeed, the $L^{1}$ stability of $B V$ weak* solutions follows directly once existence of $B V$ weak ${ }^{*}$ solutions is established, see Theorem 4.3 ,

As an illustrative example of the use of $B V$ weak* solutions, we derive the Rankine-Hugoniot jump conditions, by explicitly differentiating jumps to get Dirac masses in the derivative, and similarly express the entropy conditions. We also show that the quasilinear form of the equation is satisfied at any Lebesgue point of a weak* solution. Our derivation of the Rankine-Hugoniot conditions is both easier and more general than the usual derivation [5. As a further example, we express the solution of the Riemann problem as a Bochner integral, which in this case coincides with the Gelfand integral.

Finally, in an appendix, we consider the conditions that imply that an abstract function be G-weak differentiable. Indeed, we show that for $1<q \leq \infty$, the space $W_{w *}^{1, q}\left(0, T ; X^{*}\right)$ is isometric to the space of functions having bounded variation, introduced by Brezis in 3 .

Our goal in this paper has been to introduce the calculus of the Gelfand integral and to define the notion of weak* solution, showing that it is consistent with the usual well-known class of $B V$ weak solutions. In the upcoming papers 14. 15, 16, we develop these results, as follows. In [14, we extend the 
definition in a natural way to rigorously allow the use of Dirac masses to deal with vacuums and other discontinuities in the medium, and in 15 we implement a front-tracking scheme for approximating solutions with vacuum. In 16, we extend these ideas to an abstract and general framework in which we define weak* solutions for multi-dimensional systems of balance laws. It is hoped that the availability of a wide variety of test function spaces $X$ will shed light on the problems of instability and nonuniqueness of (strongly measurable) weak solutions.

The paper is arranged as follows. In Section 2, we recall the basic facts we need and establish notation for the rest of the paper. In Section 3 we recall the Gelfand integral and develop the calculus thereof: we do this for an abstract target space $X^{*}$, so we can use these results unchanged in more general contexts. In Section 4 we use the Gelfand integral to define weak* solutions, and prove that weak* solutions are distributional solutions, and weak solutions which satisfy appropriate growth conditions are weak* solutions. Finally, in the appendix, we consider necessary and sufficient conditions for a function to have a $\mathrm{G}$-weak derivative.

\section{Preliminaries}

We begin by setting notation and recalling various facts about functions, measures and derivatives that will be useful throughout the paper. For general references, we refer the reader to the books of Royden, Cembranos \& Mendoza, Diestel \& Uhl, etc. [18, 4, 7].

2.1. Measures and Distributions. Let $\Omega \subset \mathbb{R}$ be an open set. Denote the Borel $\sigma$-algebra on $\Omega$ by $\mathcal{B}(\Omega)$, and Lebesgue measure on the measure space $(\Omega, \mathcal{B}(\Omega))$ by $\lambda$; we also write $d x=d \lambda=\lambda(d x)$.

For any distribution $T \in \mathcal{D}^{\prime}(\Omega)$, we use the standard pairing $\langle T, \varphi\rangle$ to denote the action of $T$ on $\varphi \in \mathcal{D}(\Omega)=C_{c}^{\infty}(\Omega)$. Any integrable function $f \in L_{l o c}^{1}(\Omega)$ acts as distribution by

$$
\langle f, \varphi\rangle:=\int f \varphi d x, \quad \varphi \in \mathcal{D}(\Omega) .
$$

Let $M_{l o c}(\Omega)$ denote the set of all sigma-finite Radon measures on the measure space $(\Omega, \mathcal{B}(\Omega))$. Any measure $\nu \in M_{l o c}(\Omega)$ is also a distribution via the action

$$
\langle\nu, \varphi\rangle:=\int \varphi(x) \nu(d x)=\int \varphi(x) d \nu, \quad \varphi \in \mathcal{D}(\Omega) .
$$

We embed the integrable functions $L^{1}(\Omega)$ in the space of Radon measures by means of the natural mapping $T: L^{1}(\Omega) \rightarrow M(\Omega), T(f)=: T_{f}$, defined by

$$
T_{f}(E)=\int_{E} f(x) d x, \quad E \in \mathcal{B}(\Omega) .
$$

If $f \in L^{1}(\Omega)$, it is easy to see that $T_{f}$ is a Radon measure which satisfies $\left\|T_{f}\right\|_{M(\Omega)}=\|f\|_{L^{1}(\Omega)}$, so that $T$ is norm preserving, and we can regard

$$
L_{l o c}^{1}(\Omega) \subset M_{l o c}(\Omega) \subset \mathcal{D}^{\prime}(\Omega), \quad L^{1}(\Omega) \subset M(\Omega) \subset \mathcal{D}^{\prime}(\Omega) .
$$

Next, suppose that $F: \Omega \subset \mathbb{R} \rightarrow \mathbb{R}$, with $\Omega \subset \mathbb{R}$ open. We denote the classical derivative of $F$ at $x \in \Omega$ by $\frac{d F}{d x}$, wherever this exists. We adopt the 
convention that if $\frac{d F}{d x}$ exists $\lambda$-almost surely, then we redefine $\frac{d F}{d x}$ to be zero at all points $x$ at which the classical derivative is not defined. If $F \in L_{l o c}^{1}(\Omega)$ has a weak derivative, we denote the weak derivative by $F^{\prime}(x)$ or $\mathrm{D} F \in L_{l o c}^{1}(\Omega)$. Finally, we denote the distributional derivative of $F$ by $\mathbb{D} F \in \mathcal{D}^{\prime}(\Omega)$.

2.2. BV Functions. We recall a few basic facts about $B V$ functions. The total variation of a function $F:(a, b) \rightarrow \mathbb{R}$ is

$$
\left.\mathbb{V}(F,(a, b)):=\sup _{\mathcal{P}} \sum_{k=1}^{N} \mid F\left(x_{k+1}\right)-F\left(x_{k}\right)\right) \mid,
$$

where $\mathcal{P}:=\left\{x_{k}\right\}_{k=1}^{N}$ ranges over the set of all finite increasing sequences in $(a, b)$, and we say that $F$ is of function of bounded variation on $(a, b)$ if $\mathbb{V}(F,(a, b))<\infty$. We denote the space of functions of bounded variation by

$$
B V((a, b)):=\{F:(a, b) \rightarrow R: \mathbb{V}(F,(a, b))<\infty\} .
$$

We note that the usual convention is to treat $B V$ functions on a closed interval $[a, b]$, but we find it convenient to consider an open interval when studying their distributional derivatives. Clearly, $F$ can be extended to the closed interval $[a, b]$ by continuity. The classical derivative $\frac{d F}{d x}$ exists Lebesgue almost surely, the left and right limits $F(x \pm):=\lim _{z \rightarrow x \pm} F(z)$ exist for all $x \in(a, b)$, and the set of all jump discontinuities

$$
\mathcal{J}=\{x \in(a, b): F(x+)-F(x-) \neq 0\}
$$

is at most countable. The right-continuous modification $F^{*}$ of $F$ satisfies $F^{*}=F_{c}+F_{j}+F_{s}$, where the functions

$$
\begin{aligned}
F_{c}(x) & :=F(a)+\int_{a}^{x} \frac{d F}{d x} d s, \\
F_{j}(x) & :=\sum_{\bar{x} \in \mathcal{J}} H(x-\bar{x})(F(\bar{x}+)-F(\bar{x}-)), \quad H(x):=\mathcal{X}_{x \geq 0}, \\
F_{s}(x) & :=F^{*}(x)-F_{c}(x)-F_{j}(x),
\end{aligned}
$$

are the absolutely continuous, jump and singular continuous parts of $F$, respectively.

Each $B V$ function gives rise to a finite signed Borel measure via

$$
\nu(y, x]:=F(x+)-F(y+), \quad \forall x, y \in(a, b),
$$

and $\nu(a, b):=F(b)-F(a)$, with variation $\|\nu\|_{M(a, b)}=|\nu|(a, b)=\mathbb{V}(F,(a, b))$. Applying (77) to the decomposition (66) of $F$ yields $\nu=\nu_{c}+\nu_{j}+\nu_{s}$, where $\nu_{c}$ is absolutely continuous, $\nu_{c} \ll \lambda$, with $\lambda$-a.e. Radon-Nikodym derivative $\frac{d \nu_{c}}{d \lambda}=\frac{d F}{d x} ; \nu_{j}$ is a purely atomic singular measure $\nu_{j} \perp \lambda$; and $\nu_{s}$ is a singular continuous measure, $\nu_{s} \perp \lambda$. Moreover, we have the identities

$$
\nu_{c}(A)=\int_{A} \frac{d F_{c}}{d x} d x \quad \text { and } \quad \nu_{j}=\sum_{\bar{x} \in \mathcal{J}}(F(\bar{x}+)-F(\bar{x}-)) \delta_{\bar{x}} .
$$

These decompositions fully describe the distributional derivative of the function $F \in B V(a, b)$, as follows. 
Lemma 2.1. Using the above notation, $F \in B V(a, b)$ has distributional derivative given by

$$
\begin{aligned}
\mathbb{D} F & =\mathbb{D} F^{*}=\nu=\nu_{c}+\nu_{j}+\nu_{s}, \quad \text { with } \\
\mathbb{D} F_{c} & =\nu_{c}, \quad \mathbb{D} F_{j}=\nu_{j}, \quad \text { and } \quad \mathbb{D} F_{s}=\nu_{s}
\end{aligned}
$$

so that

$$
\begin{aligned}
\langle\mathbb{D} F, \varphi\rangle & =\int \varphi \nu_{c}(d x)+\int \varphi \nu_{j}(d x)+\int \varphi \nu_{s}(d x) \\
& =\int \varphi \frac{d F}{d x} d x+\sum_{\bar{x} \in \mathcal{J}}(F(\bar{x}+)-F(\bar{x}-)) \varphi(\bar{x})+\int \varphi \nu_{s}(d x) .
\end{aligned}
$$

2.3. Products of Banach Spaces. Let $X$ be a normed space, with norm denoted by $\|\cdot\|_{X}$. Recall that the dual $X^{*}$ of $X$ has norm

$$
\|\phi\|_{X^{*}}=\sup _{x \in X, x \neq 0} \frac{|\phi(x)|}{\|x\|_{X}}=\sup _{x \in X, x \neq 0} \frac{\langle\phi, x\rangle}{\|x\|_{X}},
$$

where $\langle\phi, x\rangle$ denotes the pairing between $\phi$ and $x$, and $X^{*}$ is a Banach space. We recall the well-known property that this pairing distinguishes elements in $X$ or $X^{*}$ : that is, if $\langle\phi, x\rangle=0$ for all $x \in X$, then $\phi=0$, and if $\langle\phi, x\rangle=0$ for all $\phi \in X^{*}$, then $x=0$.

Because we are interested in systems, our solutions will consist of vectors with values in $\left(X^{*}\right)^{n}$. For any $X$, we equip the product space $X^{n}$ with the "Euclidean" norm

$$
\|x\|_{X^{n}}:=\left(\sum_{i=1}^{n}\left\|x_{i}\right\|_{X}^{2}\right)^{1 / 2}, \quad x=\left(x_{1}, \ldots, x_{n}\right) \in X^{n} .
$$

We show that there is no ambiguity between $\left(X^{*}\right)^{n}$ and $\left(X^{n}\right)^{*}$.

Lemma 2.2. For any $n \geq 1$, the mapping $I:\left(X^{*}\right)^{n} \rightarrow\left(X^{n}\right)^{*}$, given by

$$
[I(F)](x)=\sum_{i=1}^{n} F_{i}\left(x_{i}\right) \quad \text { for } \quad F=\left(F_{1}, \ldots, F_{i}\right) \in\left(X^{*}\right)^{n}, \quad x \in X^{n},
$$

is an isometric isomorphism, $\|I(F)\|_{\left(X^{n}\right)^{*}}=\|F\|_{\left(X^{*}\right)^{n}}$.

Proof. The map $I$ is trivially injective and onto; to show it is an isometry, first note that

$$
|[I(F)](x)|=\left|\sum_{i=1}^{n} F_{i}\left(x_{i}\right)\right| \leq \sum_{i=1}^{n}\left\|F_{i}\right\|_{X^{*}}\left\|x_{i}\right\|_{X} \leq\|F\|_{\left(X^{*}\right)^{n}}\|x\|_{X^{n}},
$$

by Cauchy-Schwarz in $\mathbb{R}^{n}$, so that $\|I(F)\|_{\left(X^{n}\right)^{*}} \leq\|F\|_{\left(X^{*}\right)^{n}}$. Next, given any $F \in\left(X^{*}\right)^{n}$ and $\varepsilon>0$, we can find $\bar{x}_{i} \in X, i=1, \ldots, n$ such that each $\left\|\bar{x}_{i}\right\|=1$ and

$$
\left(\sum_{1=1}^{n}\left|F_{i}\left(\bar{x}_{i}\right)-\left\|F_{i}\right\|_{X^{*}}\right|^{2}\right)^{1 / 2} \leq \sqrt{n} \varepsilon .
$$

Now set

$$
\bar{y}=\|F\|_{\left(X^{*}\right)^{n}}^{-1}\left(\bar{x}_{1}\left\|F_{1}\right\|_{X^{*}}, \ldots, \bar{x}_{n}\left\|F_{n}\right\|_{X^{*}}\right),
$$


so that $\|\bar{y}\|_{X^{n}}=1$, and

$$
[I(F)](\bar{y})-\|F\|_{\left(X^{*}\right)^{n}}=\|F\|_{\left(X^{*}\right)^{n}}^{-1} \sum_{i=1}^{n}\left\|F_{i}\right\|_{X^{*}}\left(F_{i}\left(\bar{x}_{i}\right)-\left\|F_{i}\right\|_{X^{*}}\right) \geq-\sqrt{n} \varepsilon,
$$

again using Cauchy-Schwarz. Since $\varepsilon$ is arbitrary, the result follows.

Corollary 2.3. Let $\Omega \subset \mathbb{R}^{d}$ be an open set, and $C_{0}(\Omega)$ denote the closure of $C_{c}(\Omega)$ under the sup-norm. For $n \geq 1$, the mapping $T: M(\Omega)^{n} \rightarrow\left(C_{0}(\Omega)^{n}\right)^{*}$, given by

$$
\langle T(\mu), \varphi\rangle=\int_{\Omega} \varphi(x) \cdot \mu(d x):=\sum_{i=1}^{n} \int \varphi_{i}(x) \mu_{i}(d x),
$$

is an isometric isomorphism,

$$
\|T(\mu)\|_{\left(C_{0}(\Omega)^{n}\right)^{*}}=\|\mu\|_{M^{n}(\Omega)}=\left(\sum_{i=1}^{n}\left(\left|\mu_{i}\right|(\Omega)\right)^{2}\right)^{1 / 2} .
$$

Proof. Since $\Omega \subset \mathbb{R}^{d}$ is locally compact, the proof for $n=1$ follows from the Riesz-Markov and Riesz Representation theorems [18; the general case follows directly.

2.4. Measurability and Equivalence of Functions. Because we want to integrate in Banach spaces, we recall several notions of measurability and note the relationships between them. We follow the conventions used in the book of Diestel and Uhl [7. Unless explicitly stated otherwise, $f$ is a map of a single variable with values in a Banach space, $f:[0, T] \rightarrow X$, and the Borel $\sigma$-algebra is always assumed.

The map $f$ is measurable, or $\mathfrak{B}$-measurable, if $f^{-1}(A) \in \mathfrak{B}:=\mathcal{B}([0, T])$ for each $A \in \mathcal{B}(X)$.

The map $f(t)$ is called $\lambda$-essentially separably valued if there exists $N \in \mathfrak{B}$ with $\lambda(N)=0$ and a countable set $H \subset X$ such that $f([0, T] \backslash N) \subset \bar{H}$; $f$ is separably valued if $f([0, T]) \subset \bar{H}$.

The map $f$ is simple if there exist vectors $u_{1}, u_{2}, \ldots, u_{n} \in X$ and sets $E_{1}, E_{2}, \ldots, E_{n} \in \mathfrak{B}$ such that

$$
f(t)=\sum_{i=1}^{n} u_{i} \mathcal{X}_{E_{i}}(t)
$$

where $\mathcal{X}_{E_{i}}$ is the indicator function of $E_{i}$.

The map $f$ is $\lambda$-measurable, or strongly measurable, if there is a sequence of simple functions $f_{n}:[0, T] \rightarrow X$ such that

$$
\lim _{n \rightarrow \infty}\left\|f_{n}(t)-f(t)\right\|=0 \quad \lambda \text {-almost surely on }[0, T] \text {. }
$$

It is not hard to show that a map is $\lambda$-measurable if and only if it is both $\mathfrak{B}$-measurable and $\lambda$-essentially separably valued. In particular, if the space $X$ is separable, then $\lambda$-measurability and $\mathfrak{B}$-measurability are equivalent.

The map $f:[0, T] \rightarrow X$ is norm-measurable if its norm $\|f(t)\|:[0, T] \rightarrow \mathbb{R}$ is $\lambda$-measurable (i.e. Lebesgue measurable).

If $f:[0, T] \rightarrow X$ is $\lambda$-measurable, it is norm-measurable. Moreover, if $f_{n}:[0, T] \rightarrow X$ is a sequence of $\lambda$-measurable functions which almost surely 
converge in norm, $\left\|f_{n}(t)-f(t)\right\| \rightarrow 0$ as $n \rightarrow \infty \lambda$-almost surely on $[0, T]$, then the limit $f$ is $\lambda$-measurable.

We now define a map $f:[0, T] \rightarrow X$ to be weakly measurable, or scalar measurable, if for each $\phi \in X^{*}$, the function

$$
\phi(f(\cdot))=\langle\phi, f(\cdot)\rangle:[0, T] \rightarrow \mathbb{R}
$$

is $\lambda$-measurable, or equivalently $\mathfrak{B}$-measurable.

A measurable map is weakly measurable, and the converse holds if $f$ is $\lambda$-essentially separably valued; this is the statement of Pettis' Measurability Theorem; see [7. It follows immediately that if $X$ is separable, then $f$ is $\lambda$ measurable if and only if it is weakly measurable. Pettis's theorem also yields the fact that $f:[0, T] \rightarrow X$ is $\lambda$-measurable if and only if it is the $\lambda$-almost everywhere uniform limit of a sequence of countably valued $\lambda$-measurable functions.

The map $f:[0, T] \rightarrow X^{*}$ is weak ${ }^{*}$-measurable if for each $x \in X$, the function

$$
[f(\cdot)](x)=\langle f(\cdot), x\rangle:[0, T] \rightarrow \mathbb{R}
$$

is $\lambda$-measurable.

In view of the standard embedding $X \subset X^{* *}$, it follows that an $X^{*}$-valued weak measurable map $f:[0, T] \rightarrow X^{*}$ is weak* measurable.

We now introduce notions of equivalence of functions. Suppose we are given $f, g:[0, T] \rightarrow X$ and $f^{*}, g^{*}:[0, T] \rightarrow X^{*}$.

The maps $f$ and $g$ are $\lambda$-equivalent if $f(t)=g(t) \lambda$-a.e.

The maps $f$ and $g$ are weakly equivalent if, for all $\phi \in X^{*}$, and for $\lambda$-a.e. $t$, we have $\langle\phi, f(t)\rangle=\langle\phi, g(t)\rangle$.

The maps $f^{*}$ and $g^{*}$ are weak ${ }^{*}$ equivalent if, for all $x \in X$, and for $\lambda$-a.e. $t$, we have $\left\langle f^{*}(t), x\right\rangle=\left\langle g^{*}(t), x\right\rangle$.

It is clear that $\lambda$-equivalent functions are weakly equivalent, and that weakly equivalent functions with values in $X^{*}$ are weak* equivalent. In fact, the converse of this is true, provided the functions are strongly measurable; see $\mathbf{7}$.

Lemma 2.4. Suppose that $f, g:[0, T] \rightarrow X$ and $f^{*}, g^{*}:[0, T] \rightarrow X^{*}$ are $\lambda$-measurable. If $f$ and $g$ are weakly equivalent, then they are $\lambda$-equivalent, and if $f^{*}$ and $g^{*}$ are weak ${ }^{*}$ equivalent, then they are $\lambda$-equivalent.

In this lemma, the requirement that the functions be strongly measurable is essential. We will later consider non-measurable functions that are weak or weak* measurable, in which cases the corresponding weaker notions of equivalence are appropriate.

2.5. The Bochner Integral. The integral of a simple function, given by $h(t)=\sum_{i=1}^{N} u_{i} \mathcal{X}_{E_{i}}:[0, T] \rightarrow X$, is defined in the obvious way,

$$
\int_{0}^{T} h(t) \lambda(d t)=\int_{0}^{T} h(t) d t:=\sum_{i=1}^{N} u_{i} \lambda\left(E_{i}\right) \in X .
$$

We say that a $\lambda$-measurable function $f:[0, T] \rightarrow X$ is Bochner integrable, or summable, if there exists a sequence of simple functions $\left\{h_{n}\right\}_{n \geq 1}$ such that the Lebesgue integral $\int_{0}^{T}\left\|h_{n}-f\right\| d t \rightarrow 0$ as $n \rightarrow \infty$. It follows that if $f$ is 
summable, its integral over any $E \in \mathfrak{B}$ exists in the space $X$,

$$
\int_{E} f(t) d t:=\lim _{n \rightarrow \infty} \int_{E} h_{n}(t) d t .
$$

The calculus of the Bochner integral is well known, and many of the usual theorems of the Lebesgue integral translate directly to statements on the Bochner integral. These include a Bochner dominated convergence theorem and a Bochner-Lebesgue differentiation theorem, which states that for $\lambda$-almost all $s \in[0, T]$,

$$
\begin{aligned}
\lim _{h \rightarrow 0} \frac{1}{h} \int_{s}^{s+h}\|f(t)-f(s)\| d t & =0 \quad \text { and } \\
\lim _{h \rightarrow 0} \frac{1}{h} \int_{s}^{s+h} f(t) d t & =f(s) .
\end{aligned}
$$

Hille's theorem states that Bochner integration commutes with closed linear operators, and in particular implies that if $f:[0, T] \rightarrow X$ and $f^{*}:[0, T] \rightarrow X^{*}$ are Bochner integrable, then for each $E \in \mathfrak{B}, x \in X$ and $\phi \in X^{*}$,

$$
\begin{aligned}
\left\langle\phi, \int_{E} f(t) d t\right\rangle & =\int_{E}\langle\phi, f(t)\rangle d t \quad \text { and } \\
\left\langle\int_{E} f^{*}(t) d t, x\right\rangle & =\int_{E}\left\langle f^{*}(t), x\right\rangle d t .
\end{aligned}
$$

The Bochner integral allows us to introduce $L^{p}$ spaces as a natural generalization of the usual space $L^{p}(0, T)$ of real valued functions. For each $1 \leq p \leq \infty$, the Banach space $L^{p}(0, T ; X)$ consists of all $\lambda$-equivalence classes of Bochner integrable functions $f:[0, T] \rightarrow X$ such that $\|f\|_{L^{p}(0, T ; X)}<\infty$, where

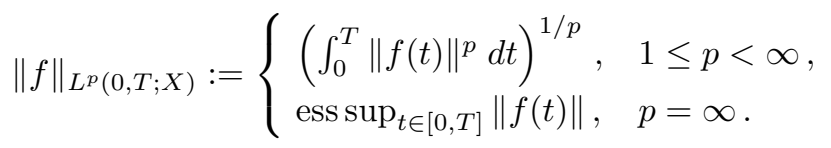

Now let $X, Y$ be Banach spaces with $Z=X \cap Y$ nonempty, and suppose $f \in L^{1}(0, T ; X)$. We say that $g \in L^{1}(0, T ; Y)$ is the Bochner weak derivative or B-weak derivative of $f$, written $f^{\prime}(t)=g(t)$, provided that $f, g$ are Bochner integrable as functions from $[0, T] \rightarrow Z$ and

$$
\int_{0}^{T} f(t) \varphi^{\prime}(t) d t=-\int_{0}^{T} g(t) \varphi(t) d t
$$

for all scalar functions $\varphi \in C_{c}^{\infty}(0, T)$. For $X^{*}$-valued functions, B-weak derivatives are fully determined by their actions as functionals: that is, for $f, g \in L^{1}\left(0, T ; X^{*}\right), f^{\prime}=g$ if and only if for every $x \in X$ and $\varphi \in C_{c}^{\infty}(0, T)$,

$$
\int_{0}^{T}\langle f(t), x\rangle \varphi^{\prime}(t) d t=-\int_{0}^{T}\langle g(t), x\rangle \varphi(t) d t .
$$

For $1 \leq p \leq \infty$, the Sobolev space $W^{1, p}(0, T ; X)$ is the set of all functions $f \in L^{p}(0, T ; X)$ with $f^{\prime} \in L^{p}(0, T ; X)$, with norm

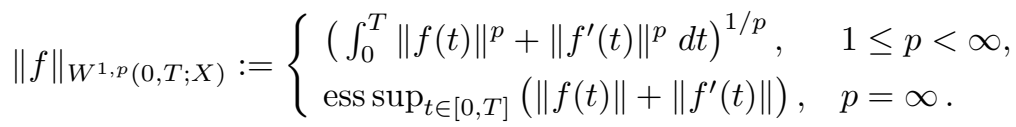


For our purposes, the most important feature of functions in $W^{1, p}(0, T ; X)$ is the Fundamental Theorem of Calculus, which follows from the fact that if $f \in L^{p}(0, T ; X)$, the integral $\int_{0}^{t} f(s) d s$ is absolutely continuous as a function of $t$.

Theorem 2.5. Let $f \in W^{1, p}(0, T ; X)$ for some $1 \leq p \leq \infty$, and define $\bar{f}(t)=f(0)+\int_{0}^{t} f^{\prime}(s) d s$. Then $\bar{f} \in C([0, T] ; X)$ is a.e. differentiable and $\bar{f}=f$ a.e.. The function $g:[0, T] \rightarrow X$ given by

$$
g(s):= \begin{cases}\lim _{h \rightarrow 0} \frac{1}{h}(\bar{f}(s+h)-\bar{f}(s)), & \text { if the limit exists, } \\ 0, & \text { otherwise, }\end{cases}
$$

is strongly measurable and satisfies $g(t)=f^{\prime}(t) \lambda$-a.e. on $[0, T]$. If $p=\infty$, then $\bar{f}$ is Lipschitz continuous with

$$
\|\bar{f}(t)-\bar{f}(s)\| \leq L|t-s| \quad \text { with } \quad L:=\operatorname{esssup}_{t \in[0, T]}\left\|f^{\prime}(t)\right\| .
$$

The following form of Hölder's inequality holds: if $f \in L^{p}(0, T ; X)$ and $g^{*} \in L^{q}\left(0, T ; X^{*}\right), p^{-1}+q^{-1}=1$, then $\left\langle g^{*}, f\right\rangle:[0, T] \rightarrow \mathbb{R}$ is integrable and satisfies

$$
\int_{0}^{T}\left|\left\langle g^{*}(s), f(s)\right\rangle\right| d s \leq\|f\|_{L^{p}(0, T ; X)}\left\|g^{*}\right\|_{L^{q}\left(0, T ; X^{*}\right)} .
$$

As a consequence, we can integrate by parts: given $f \in W^{1, p}(0, T ; X)$ and $g^{*} \in W^{1, q}\left(0, T ; X^{*}\right)$, we have $\left\langle g^{*}, f\right\rangle \in W^{1,1}(0, T)$, and

$$
\int_{0}^{T}\left\langle g^{* \prime}(t), f(t)\right\rangle d t=\left.\left\langle\bar{g}^{*}(s), \bar{f}(s)\right\rangle\right|_{0} ^{T}-\int_{0}^{T}\left\langle g^{*}(t), f^{\prime}(t)\right\rangle d t
$$

where $\bar{f}, \bar{g}$ are the continuous representatives of $f$ and $g$, respectively.

\section{The Gelfand Integral}

It is sometimes too restrictive to consider only the Bochner integral of strongly measurable functions with values in a Banach space. Here we recall the Gelfand integral, which is better suited to our purposes. Throughout this section we fix a Banach space $X$ and let $X^{*}$ denote its dual. The Gelfand integral is similar to the Dunford integral, but takes its values in the space $X^{*}$. For a detailed discussion see Tulcea and Tulcea [22, Diestel and Uhl [7, or Cembranos and Mendoza [4].

Suppose that we are given a weak*-measurable function $\Phi:[0, T] \rightarrow X^{*}$, and suppose also that

$$
\langle\Phi(\cdot), x\rangle \in L^{1}(0, T) \quad \text { for all } \quad x \in X .
$$

For a given $E \in \mathfrak{B}=\mathcal{B}([0, T])$, we define the map $T_{E}: X \rightarrow L^{1}(0, T)$ by

$$
T_{E}(x)=\langle\Phi(\cdot), x\rangle \chi_{E}(\cdot) \in L^{1}(0, T) .
$$

It is clear that $T_{E}$ is linear, and if $x_{n} \rightarrow x$ and $T_{E}\left(x_{n}\right) \rightarrow y$ in $L^{1}$, then by the Riesz-Fischer theorem, a subsequence $T_{E}\left(x_{n_{k}}\right)(s) \rightarrow y(s)$ a.e., while also $T_{E}\left(x_{n}\right)(s) \rightarrow\langle\Phi(s), x\rangle \chi_{E}(s)$ for all $s \in[0, T]$. It follows that $y \in L^{1}(0, T)$, so $T_{E}$ is closed, and further, by the closed graph theorem, it is bounded, so we 
can write $\left\|T_{E}(x)\right\|_{L^{1}} \leq\left\|T_{E}\right\|\|x\|$ for all $x \in X$. Since integration is a bounded linear operator of $L^{1}$ into $\mathbb{R}$, it follows that the map

$$
x \mapsto \int_{0}^{T} T_{E}(x)(s) d s=\int_{E}\langle\Phi(s), x\rangle d s
$$

is a bounded linear functional on $X$, so defines an element of the dual $X^{*}$.

Definition 3.1. Let $\Phi:[0, T] \rightarrow X^{*}$ be a weak $k^{*}$ measurable function such that $\langle\Phi(\cdot), x\rangle \in L^{1}(0, T)$ for every $x \in X$. The Gelfand integral of $\Phi$ over measurable set $E \subset(0, T)$, denoted by $\star \int_{E} \Phi(s) d s$, is an element of $X^{*}$ defined by

$$
\left\langle\star \int_{E} \Phi(s) d s, x\right\rangle=\int_{E}\langle\Phi(s), x\rangle d s \quad \text { for all } \quad x \in X .
$$

3.1. The Spaces $L_{w *}^{q}\left(0, T ; X^{*}\right)$. It is clear from (11) that if two weak*measurable functions are weak*-equivalent, then their Gelfand integrals coincide. We are thus led to consider equivalence classes,

$$
[\Psi]=\left\{\widetilde{\Psi}:[0, T] \rightarrow X^{*}: \Psi \text { and } \widetilde{\Psi} \text { are weakly*-equivalent }\right\} .
$$

We now wish to describe the sets of (equivalence classes of) weak*-measurable functions which are $L^{q}$ Gelfand integrable, and to give an appropriate norm. Note that even if $\Phi:[0, T] \rightarrow X^{*}$ is weak*-measurable, in general its norm $\|\Phi\|:[0, T] \rightarrow \mathbb{R}$ is not measurable. However, the theory of liftings provides the following lemma, $\mathbf{4}, \mathbf{2 2}]$.

Lemma 3.2. Each Gelfand integrable function $\Psi:[0, T] \rightarrow X^{*}$ is weak*equivalent to a map $\hat{\Psi}:[0, T] \rightarrow X^{*}$ whose norm $\|\hat{\Psi}\|:[0, T] \rightarrow \mathbb{R}$ is measurable. Moreover, if $\Psi_{1}$ and $\Psi_{2}$ are weak ${ }^{*}$-equivalent and norm-measurable, then $\left\|\Psi_{1}\right\|=\left\|\Psi_{2}\right\|$ almost everywhere.

It is easy to show that if $X$ is separable, all weak*-measurable functions are norm-measurable.

We are now in a position to describe the $X^{*}$ valued Gelfand $L^{q}$ spaces, for $1 \leq q \leq \infty$. Given an equivalence class $[\Psi]$ of Gelfand integrable functions, set

$$
\|[\Psi]\|_{q}:=\inf \left\{\|g\|_{L^{q}(0, T)}:\|\hat{\Psi}(t)\| \leq g(t) \lambda \text {-a.e. }\right\},
$$

where $\hat{\Psi} \in[\Psi]$ is a norm-measurable element of the equivalence class. It follows that $\|\cdot\|_{q}$ is a norm, and we let $L_{w *}^{q}\left(0, T ; X^{*}\right)$ be the space of equivalence classes $[\Psi]$ of finite norm,

$$
L_{w *}^{q}\left(0, T ; X^{*}\right):=\left\{[\Psi]:\|[\Psi]\|_{q}<\infty\right\} .
$$

It is not difficult to show that $L_{w *}^{q}\left(0, T ; X^{*}\right)$ is a Banach space and that the trivial inclusion

$$
L^{q}\left(0, T ; X^{*}\right) \subset L_{w *}^{q}\left(0, T ; X^{*}\right) \quad \text { via } \quad f \mapsto[f],
$$

is a norm-preserving isomorphism. In particular, a Bochner integrable map $f:[0, T] \rightarrow X^{*}$ is also Gelfand integrable, and when they both exist, the integrals coincide. Also, if $\hat{\Psi} \in[\Psi] \in L_{w *}^{q}\left(0, T ; X^{*}\right)$ is norm-measurable, then $\|\hat{\Psi}\| \in L^{q}(0, T)$ and

$$
\|[\Psi]\|_{q}=\|\| \hat{\Psi}(\cdot)\|\|_{L^{q}(0, T)} .
$$


Similarly,

$$
\int_{0}^{T}|\langle\hat{\Psi}(t), x\rangle|^{q} d t \leq \int_{0}^{T}\|\hat{\Psi}(t)\|^{q}\|x\|^{q} d t=\|[\Psi]\|_{q}^{q}\|x\|^{q},
$$

and we conclude that for any $x \in X$ and $\widetilde{\Psi} \in[\Psi] \in L_{w *}^{q}\left(0, T ; X^{*}\right)$, we have

$$
\langle\widetilde{\Psi}(\cdot), x\rangle \in L^{q}(0, T) \quad \text { with } \quad\|\langle\widetilde{\Psi}(\cdot), x\rangle\|_{L^{q}(0, T)} \leq\|[\Psi]\|\left\|_{q}\right\| x \| .
$$

It turns out that $L_{w *}^{q}\left(0, T ; X^{*}\right)$ is the dual space of $L^{p}(0, T ; X)$, provided $1 \leq p<\infty$ and $p^{-1}+q^{-1}=1$; see $[4$ for details.

Theorem 3.3. For each $\widetilde{\Psi} \in[\Psi] \in L_{w *}^{q}\left(0, T ; X^{*}\right)$ and $f \in L^{p}(0, T ; X)$, the function

$$
\langle\widetilde{\Psi}(\cdot), f(\cdot)\rangle \in L^{1}(0, T),
$$

and is independent of representative $\widetilde{\Psi}$. Moreover, the natural linear map $[\Psi] \rightarrow I([\Psi]) \in L^{p}(0, T ; X)^{*}$, given by

$$
\langle I[\Psi], f\rangle:=\int_{0}^{T}\langle\widetilde{\Psi}(s), f(s)\rangle d s, \quad f \in L^{p}(0, T ; X),
$$

is an isometric isomorphism of $L_{w *}^{q}\left(0, T ; X^{*}\right)$ onto $L^{p}(0, T ; X)^{*}$.

3.2. Absolute Continuity. Although the spaces $L_{w *}^{q}\left(0, T ; X^{*}\right)$ can be regarded as well known, to the authors' knowledge the calculus of Gelfand integrable functions has not previously been developed. Here we develop this calculus in parallel with the well-known calculus of the Bochner integral. We begin by proving absolute continuity of the Gelfand integral.

Theorem 3.4. Fix $1 \leq q \leq \infty$, and suppose $[\Phi] \in L_{w *}^{q}\left(0, T ; X^{*}\right)$ with $\widetilde{\Phi} \in[\Phi]$. The linear map $\Psi(t):[0, T] \rightarrow X^{*}$ defined by

$$
\begin{aligned}
\Psi(t) & =\star \int_{0}^{t} \widetilde{\Phi}(s) d s, \quad \text { that is } \\
\langle\Psi(t), x\rangle & =\int_{0}^{t}\langle\widetilde{\Phi}(s), x\rangle d s, \quad x \in X,
\end{aligned}
$$

is absolutely continuous. Also, the total variation function $\mathbb{V}_{\Psi}:[0, T] \rightarrow \mathbb{R}$, defined by

$$
\mathbb{V}_{\Psi}(t):=\mathbb{V}(\Psi,[0, t])=\sup _{\mathcal{P}_{[0, t]}} \sum\left\|\Psi\left(t_{n}\right)-\Psi\left(t_{n-1}\right)\right\|,
$$

the supremum being over partitions $\mathcal{P}_{[0, t]}=\left\{0=t_{0}<t_{1} \cdots<t_{n}=t\right\}$, is absolutely continuous, with pointwise derivative $\frac{d \mathbb{V}_{\Psi}}{d t} \in L^{q}(0, T)$, and we have

$$
\|\Psi(t)-\Psi(s)\| \leq \mathbb{V}_{\Psi}(t)-\mathbb{V}_{\Psi}(s)=\int_{s}^{t} \frac{d \mathbb{V}_{\Psi}}{d t}(\tau) d \tau \leq \int_{s}^{t}\|\hat{\Phi}(\tau)\| d \tau
$$

for all $0 \leq s \leq t \leq T$, for $\hat{\Phi} \in[\Phi]$ norm-measurable. 
Proof. Suppose that $\hat{\Phi} \in[\Phi]$ is norm-measurable, and let $E \subset[0, T]$ be a union of disjoint intervals, $E=\bigcup_{n=1}^{N}\left(a_{n}, b_{n}\right)$. Then $\|\hat{\Phi}\| \in L^{1}(0, T)$, and we have

$$
\begin{aligned}
\sum_{n=1}^{N}\left\|\Psi\left(b_{n}\right)-\Psi\left(a_{n}\right)\right\| & =\sum_{n=1}^{N} \sup _{\|x\|=1}\left\langle\Psi\left(b_{n}\right)-\Psi\left(a_{n}\right), x\right\rangle \\
& =\sum_{n=1}^{N} \sup _{\|x\|=1} \int_{a_{n}}^{b_{n}}\langle\hat{\Phi}(s), x\rangle d s \\
& \leq \sum_{n=1}^{N} \int_{a_{n}}^{b_{n}}\|\hat{\Phi}(s)\| d s=\int_{E}\|\hat{\Phi}(s)\| d s
\end{aligned}
$$

and absolute continuity follows from that of the Lebesgue integral. Similarly, for any $0 \leq s \leq t \leq T$,

$$
\mathbb{V}_{\Psi}(t)-\mathbb{V}_{\Psi}(s) \leq \int_{s}^{t}\|\hat{\Phi}(\tau)\| d \tau
$$

so $\mathbb{V}_{\Psi}(t)$ is absolutely continuous. Thus the derivative $\frac{d \mathbb{V}_{\Psi}}{d t}$ is defined $\lambda$-a.e., and

$$
\mathbb{V}_{\Psi}(t)-\mathbb{V}_{\Psi}(s)=\int_{s}^{t} \frac{d \mathbb{V}_{\Psi}}{d t}(\tau) d \tau \quad \text { for all } \quad 0 \leq s \leq t \leq T .
$$

It follows that $0 \leq \frac{d \mathbb{V}_{\Psi}}{d t} \leq\|\hat{\Phi}(t)\| \lambda$-a.e. on $[0, T]$, and thus $\frac{d \mathbb{V}_{\Psi}}{d t} \in L^{q}(0, T)$.

The Lebesgue Differentiation Theorem implies that for all $x \in X$,

$$
\lim _{h \rightarrow 0} \frac{1}{h} \int_{t}^{t+h}\langle\widetilde{\Phi}(s), x\rangle d s=\langle\widetilde{\Phi}(t), x\rangle \quad \lambda \text {-a.e. . }
$$

Our next task is to extend this to get a Gelfand-Lebesgue Differentiation Theorem. Fix $1 \leq q \leq \infty$ and $p^{-1}+q^{-1}=1$.

Theorem 3.5. Suppose that $[\Phi] \in L_{w *}^{q}\left(0, T ; X^{*}\right)$ and $\widetilde{\Phi} \in[\Phi]$. Then for each $f \in L^{p}(0, T ; X)$,

$$
\begin{array}{ll}
\lim _{h \rightarrow 0} \frac{1}{h} \int_{t}^{t+h}\langle\widetilde{\Phi}(s), f(t)\rangle d s=\langle\widetilde{\Phi}(t), f(t)\rangle & \lambda \text {-a.e. }, \quad \text { and } \\
\lim _{h \rightarrow 0} \frac{1}{h} \int_{t}^{t+h}\langle\widetilde{\Phi}(s), f(s)\rangle d s=\langle\widetilde{\Phi}(t), f(t)\rangle \quad \lambda \text {-a.e. . }
\end{array}
$$

If $X$ is separable then for almost all $t \in(0, T)$

$$
\frac{1}{h} \star \int_{t}^{t+h} \widetilde{\Phi}(s) d s \quad \stackrel{w *}{\longrightarrow} \widetilde{\Phi}(t) \quad \text { as } \quad h \rightarrow 0 .
$$

Proof. Since $f \in L^{p}(0, T ; X)$, it is strongly measurable, and without loss of generality we may assume that it is separably valued. Let $\left\{x_{n}\right\}$ be a countable dense subset of $f([0, T])$ and let $\hat{\Phi} \in[\Phi]$ be norm-measurable. Define the set

$$
\begin{aligned}
E:=\left\{t: \frac{1}{h} \int_{t}^{t+h}\|\hat{\Phi}(s)\| d s \rightarrow\|\hat{\Phi}(t)\|\right\} & \\
& \bigcap \bigcap_{n=1}^{\infty}\left\{t: \frac{1}{h} \int_{t}^{t+h}\left\langle\widetilde{\Phi}(s), x_{n}\right\rangle d s \rightarrow\left\langle\widetilde{\Phi}(t), x_{n}\right\rangle\right\}
\end{aligned}
$$


where the limits are taken as $h \rightarrow 0$. By the Lebesgue Differentiation Theorem, $E$ has full measure, $\lambda([0, T] \backslash E)=0$. For $t \in E$ and any $n$, we have

$$
\begin{aligned}
\left|\frac{1}{h} \int_{t}^{t+h}\langle\widetilde{\Phi}(s), f(t)\rangle d s-\langle\widetilde{\Phi}(t), f(t)\rangle\right| \\
\leq\left|\frac{1}{h} \int_{t}^{t+h}\left\langle\widetilde{\Phi}(s), f(t)-x_{n}\right\rangle d s\right| \\
\quad+\left|\frac{1}{h} \int_{t}^{t+h}\left\langle\widetilde{\Phi}(s), x_{n}\right\rangle d s-\left\langle\widetilde{\Phi}(t), x_{n}\right\rangle\right|+\left|\left\langle\widetilde{\Phi}(t), x_{n}-f(t)\right\rangle\right| \\
\leq\left|\frac{1}{h} \int_{t}^{t+h}\|\hat{\Phi}(s)\| d s\right|\left\|f(t)-x_{n}\right\| \\
\quad+\left|\frac{1}{h} \int_{t}^{t+h}\left\langle\widetilde{\Phi}(s), x_{n}\right\rangle d s-\left\langle\widetilde{\Phi}(t), x_{n}\right\rangle\right|+\|\widetilde{\Phi}(t)\|\left\|x_{n}-f(t)\right\| .
\end{aligned}
$$

Since $t \in E$ and $\left\{x_{n}\right\}$ is dense in $f([0, T])$, the right hand side can be made arbitrarily small, and (14) follows.

Now set $B_{n}=\{t:\|\hat{\Phi}(t)\|<n\}$. Clearly $B_{n}$ is nonempty for all $n$ beyond some $N \geq 1$, and $\bigcup_{n} B_{n}$ has full measure. For any $n \geq N$ and $t \in E \bigcap B_{n}$, using (9), we get

$$
\begin{aligned}
\limsup _{h \rightarrow 0} \mid \frac{1}{h} \int_{t}^{t+h} & \langle\widetilde{\Phi}(s), f(s)-f(t)\rangle d s \mid \\
& \leq \limsup _{h \rightarrow 0}\left|\frac{1}{h} \int_{t}^{t+h}\|\hat{\Phi}(s)\|\|f(s)-f(t)\| d s\right| \\
& \leq n \limsup _{h \rightarrow 0}\left|\frac{1}{h} \int_{t}^{t+h}\|f(s)-f(t)\| d s\right|=0,
\end{aligned}
$$

and thus (15) follows from (14).

Now suppose that $X$ is separable and let $\left\{x_{n}\right\}_{n>1}$ be dense in $X$. Define $E$ as above and fix $t \in E$. Then there is some $\delta>0$ such that for all $0<|h|<\delta$,

$$
\frac{1}{h} \int_{t}^{t+h}\|\hat{\Phi}(s)\| d s \leq\|\hat{\Phi}(t)\|+1 .
$$

For each such $h$, define $\phi_{t}(h) \in X^{*}$ by

$$
\phi_{t}(h):=\frac{1}{h} \star \int_{t}^{t+h} \widetilde{\Phi}(s) d s,
$$

so that, for every $x \in X$,

$$
\left|\left\langle\phi_{t}(h), x\right\rangle\right| \leq \frac{1}{h} \int_{t}^{t+h}\|\hat{\Phi}(s)\| d s\|x\| \leq(\|\hat{\Phi}(t)\|+1)\|x\|,
$$

which yields $\left\|\phi_{t}(h)\right\|_{X^{*}} \leq\|\hat{\Phi}(t)\|+1$ for each $0<|h|<\delta$. Now for any fixed $x \in X$ and each $n$, we have

$$
\begin{aligned}
\left|\left\langle\phi_{t}(h)-\widetilde{\Phi}(t), x\right\rangle\right| & \leq\left|\left\langle\phi_{t}(h)-\widetilde{\Phi}(t), x_{n}\right\rangle\right|+\left|\left\langle\phi_{t}(h), x-x_{n}\right\rangle\right|+\left|\left\langle\widetilde{\Phi}(t), x_{n}-x\right\rangle\right| \\
& \leq\left|\left\langle\phi_{t}(h)-\widetilde{\Phi}(t), x_{n}\right\rangle\right|+(\|\hat{\Phi}(t)\|+1+\|\widetilde{\Phi}(t)\|)\left\|x-x_{n}\right\|,
\end{aligned}
$$


and since $t \in E$, for every $n \geq 1$ we have

$$
\limsup _{h \rightarrow 0}\left|\left\langle\phi_{t}(h)-\widetilde{\Phi}(t), x\right\rangle\right| \leq(\|\hat{\Phi}(t)\|+1+\|\widetilde{\Phi}(t)\|)\left\|x-x_{n}\right\| .
$$

Since $\left\{x_{n}\right\}$ is dense, we have $\left\langle\phi_{t}(h), x\right\rangle \rightarrow\langle\widetilde{\Phi}(t), x\rangle$, and since $x$ is arbitrary, the result follows.

3.3. The Spaces $W_{w *}^{1, q}\left(0, T ; X^{*}\right)$. Suppose that $\Psi, \Phi:[0, T] \rightarrow X^{*}$ are weak* measurable, and $[\Psi],[\Phi] \in L_{w *}^{1}\left(0, T ; X^{*}\right)$. We say that $\Phi$ is the Gelfand weak derivative or G-weak derivative of $\Psi$, written $\Psi^{\prime}(t)=\Phi(t)$ or $[\Phi]=\left[\Psi^{\prime}\right]$, if

$$
\begin{aligned}
\star \int_{0}^{T} \Psi(t) \varphi^{\prime}(t) d t & =-\star \int_{0}^{T} \Phi(t) \varphi(t) d t, \quad \text { that is } \\
\int_{0}^{T}\langle\Psi(t), x\rangle \varphi^{\prime}(t) d t & =-\int_{0}^{T}\langle\Phi(t), x\rangle \varphi(t) d t \quad \text { for all } \quad x \in X,
\end{aligned}
$$

for all scalar functions $\varphi \in C_{c}^{\infty}(0, T)$.

We now define the space $W_{w *}^{1, q}\left(0, T ; X^{*}\right)$, for $1 \leq q \leq \infty$, to be the set of weak* equivalence classes $[\Psi] \in L_{w^{*}}^{q}\left(0, T ; X^{*}\right)$ having G-weak derivative $\left[\Psi^{\prime}\right] \in L_{w *}^{q}(0, T ; X)$, with norm

$$
\|[\Psi]\|_{W_{w *}^{1, p}(0, T ; X)}:= \begin{cases}\left(\int_{0}^{T}\left(\|\hat{\Psi}(t)\|^{q}+\left\|\hat{\Psi}^{\prime}(t)\right\|^{q}\right) d t\right)^{1 / q}, & 1 \leq q<\infty \\ {\operatorname{ess} \sup _{t \in[0, T]}\left(\|\hat{\Psi}(t)\|+\left\|\hat{\Psi}^{\prime}(t)\right\|\right),} q=\infty,\end{cases}
$$

for $\hat{\Psi} \in[\Psi], \hat{\Psi}^{\prime} \in\left[\Psi^{\prime}\right]$ norm-measurable.

We are now in a position to state the Gelfand Fundamental Theorem of Calculus.

Theorem 3.6. Let $\Psi:[0, T] \rightarrow X^{*}$ be weak* measurable and let $1 \leq$ $q \leq \infty$ be given. We have $[\Psi] \in W_{w *}^{1, q}\left(0, T ; X^{*}\right)$ if and only if there exist $[\Phi] \in L_{w *}^{q}\left(0, T ; X^{*}\right)$ and $\psi_{0} \in X^{*}$ such that the mapping

$$
\widetilde{\Psi}:[0, T] \rightarrow X^{*} \quad \text { given by } \quad \widetilde{\Psi}(t):=\psi_{0}+\star \int_{0}^{t} \Phi(s) d s
$$

satisfies $\widetilde{\Psi} \in[\Psi]$.

Moreover, if $[\Psi] \in W_{w *}^{1, q}\left(0, T ; X^{*}\right)$, then there exists an absolutely continuous representative $\bar{\Psi} \in[\Psi]$ such that

$$
\bar{\Psi}(t)=\bar{\Psi}(0)+\star \int_{0}^{t} \Psi^{\prime}(s) d s .
$$

The map $\bar{\Psi}$ belongs to $L^{q}\left(0, T ; X^{*}\right)$ and has variation $\mathbb{V}_{\bar{\Psi}}$ which satisfies (13).

Proof. First, suppose that $\widetilde{\Psi} \in[\Psi]$ satisfies (17). Then for every $x \in X$, $t \in[0, T]$, we have

$$
\langle\widetilde{\Psi}(t), x\rangle=\left\langle\psi_{0}, x\right\rangle+\int_{0}^{t}\langle\Phi(s), x\rangle d s \in \mathbb{R},
$$

so the function $t \mapsto\langle\widetilde{\Psi}(t), x\rangle$ is in $W^{1, q}(0, T)$, with weak derivative $t \mapsto$ $\langle\Phi(t), x\rangle$. Thus (16) holds for any $\varphi \in C_{c}^{\infty}(0, T)$, so $[\widetilde{\Psi}] \in W_{w *}^{1, q}\left(0, T ; X^{*}\right)$ with $[\Phi]=\left[\widetilde{\Psi}^{\prime}\right]$. 
Now suppose that $[\Psi] \in W_{w *}^{1, q}\left(0, T ; X^{*}\right)$ and let $\hat{\Psi} \in[\Psi]$ and $\hat{\Phi} \in\left[\Psi^{\prime}\right]$ be norm-measurable. We need to find the trace $\psi_{0}$ of $\Psi$ at $t=0$. For each $x \in X$ and $\varphi \in C_{c}^{\infty}(0, T)$, we have

$$
\int_{0}^{T}\langle\hat{\Psi}(t), x\rangle \varphi^{\prime}(t) d t=-\int_{0}^{T}\langle\hat{\Phi}(t), x\rangle \varphi(t) d t,
$$

and (12) implies $\langle\hat{\Psi}(\cdot), x\rangle \in W^{1, q}(0, T)$ with weak derivative $\langle\hat{\Phi}(\cdot), x\rangle$. This implies that for each $x \in X$, there exists a unique absolutely continuous realvalued function $z_{x}:[0, T] \rightarrow \mathbb{R}$ such that

$$
\begin{aligned}
& z_{x}(t)=\langle\hat{\Psi}(t), x\rangle \quad \lambda \text {-a.e. } t \in[0, T], \quad \text { and } \\
& z_{x}(t)=z_{x}(0)+\int_{0}^{t}\langle\hat{\Phi}(s), x\rangle d s \quad \text { for all } t \in[0, T],
\end{aligned}
$$

where $z_{x}(0)$ is the trace of the map $t \rightarrow\langle\hat{\Psi}(t), x\rangle$ at $t=0$. Note that $z_{x}(0)$ need not equal $\langle\hat{\Psi}(0), x\rangle$, and we must show the existence of $\psi_{0} \in X^{*}$ such that $z_{x}(0)=\left\langle\psi_{0}, x\right\rangle$ for all $x \in X$.

From linearity of $\hat{\Psi}(\cdot)$, continuity of $z_{x}(\cdot)$, and (19) it follows that, for any $c_{1}, c_{2} \in \mathbb{R}$ and $x_{1}, x_{2} \in X$, we have

$$
z_{c_{1} x_{1}+c_{2} x_{2}}(t)=c_{1} z_{x_{1}}(t)+c_{2} z_{x_{2}}(t) \text { for all } t \in[0, T],
$$

so the map $X \ni x \rightarrow z_{x}(0)$ is a linear functional. Next, for each $x \in X$, (19) implies that

$$
\int_{0}^{T}\langle\hat{\Psi}(t), x\rangle d t=\int_{0}^{T} z_{x}(t) d t=z_{x}(0) T+\int_{0}^{T}\left(\int_{0}^{t}\langle\hat{\Phi}(s), x\rangle d s\right) d t .
$$

Since $t \rightarrow\|\hat{\Psi}(t)\|$ and $t \rightarrow\|\hat{\Phi}(t)\|$ are measurable and integrable, we conclude that

$$
\left|z_{x}(0)\right| \leq \frac{1}{T}\left(\int_{0}^{T}\|\hat{\Psi}(t)\| d t+T \int_{0}^{T}\|\hat{\Phi}(t)\| d t\right)\|x\| .
$$

Thus $X \ni x \rightarrow z_{x}(0)$ is also bounded, and hence there is some $\psi_{0} \in X^{*}$ such that $z_{x}(0)=\left\langle\psi_{0}, x\right\rangle$ for all $x \in X$.

It follows from (19) and Theorem 3.4 that the map $\bar{\Psi}:[0, T] \rightarrow X^{*}$, defined by

$$
\bar{\Psi}(t)=\psi_{0}+\star \int_{0}^{t} \hat{\Phi}(s) d s
$$

is absolutely continuous with G-weak derivative $\hat{\Phi}$, and so $[\bar{\Psi}] \in W_{w *}^{1, q}\left(0, T ; X^{*}\right)$. Moreover, since $\langle\bar{\Psi}(t), x\rangle=z_{x}(t)$, we have $\bar{\Psi} \in[\Psi]$. The rest of the theorem follows directly from Theorem 3.4

To simplify notation in the sequel, we adopt the convention that, unless otherwise specified, we identify $[\Phi] \in L_{w *}^{q}\left(0, T ; X^{*}\right)$ with a norm-measurable representative $\hat{\Phi}$, and if $[\Psi] \in W_{w *}^{1, q}\left(0, T ; X^{*}\right)$, we identify $[\Psi]$ with its absolutely continuous representative $\bar{\Psi}$ and $\left[\Psi^{\prime}\right]$ with a norm-measurable representative $\hat{\Psi}^{\prime}$.

Corollary 3.7. Suppose that $u \in W_{w *}^{1, q}\left(0, T ; X^{*}\right)$ with continuous representative $\bar{u}$. If there exists a strongly measurable $f:[0, T] \rightarrow X^{*}$ such that $f \in\left[u^{\prime}\right]$, then $\bar{u}$ is strongly measurable and $\bar{u} \in W^{1, q}\left(0, T ; X^{*}\right)$. 
Proof. Since $f \in\left[u^{\prime}\right]$ and $\bar{u}$ is continuous, we have

$$
\bar{u}(t)=\bar{u}(0)+\star \int_{0}^{t} f(s) d s, \quad t \in[0, T] .
$$

Moreover, since $f$ is strongly measurable, it is norm-measurable, and

$$
\int_{0}^{T}\|f(t)\|^{q} d t=\left\|\left[u^{\prime}\right]\right\|_{q}^{q}<\infty
$$

so that $f \in L^{q}\left(0, T ; X^{*}\right)$, (20) is a Bochner integral, and $\bar{u} \in W^{1, q}\left(0, T ; X^{*}\right)$.

Our next calculus theorem is an integration by parts formula. Let $1 \leq p \leq$ $\infty$ satisfy $\frac{1}{p}+\frac{1}{q}=1$, and let $X$ be a Banach space with dual $X^{*}$.

Theorem 3.8. Suppose that $f \in W^{1, p}(0, T ; X)$ and $\Psi \in W_{w *}^{1, q}\left(0, T ; X^{*}\right)$. The function $\langle\Psi(t), f(t)\rangle:[0, T] \rightarrow \mathbb{R}$ is in $W^{1,1}(0, T)$, with weak derivative

$$
\mathrm{D}\langle\Psi(t), f(t)\rangle=\left\langle\Psi^{\prime}(t), f(t)\right\rangle+\left\langle\Psi(t), f^{\prime}(t)\right\rangle \quad \lambda \text {-a.e. }
$$

where $\Psi^{\prime}$ and $f^{\prime}$ are the $G$-weak and B-weak derivatives of $\Psi$ and $f$, respectively. Moreover,

$$
\int_{0}^{T}\left\langle\Psi^{\prime}(t), f(t)\right\rangle d t=\left.\langle\bar{\Psi}(s), \bar{f}(s)\rangle\right|_{0} ^{T}-\int_{0}^{T}\left\langle\Psi(t), f^{\prime}(t)\right\rangle d t
$$

where $\bar{f}$ and $\bar{\Psi}$ are the continuous representatives of $f$ and $\Psi$, respectively.

Proof. Set $z(t)=\langle\Psi(t), f(t)\rangle$, which is in $L^{1}(0, T)$ by Theorem 3.3, assume that $f$ and $\Psi$ are continuous representatives, and fix representatives $f^{\prime}$ and norm-measurable $\Psi^{\prime}$. Let $E=\bigcup_{n=1}^{N}\left(a_{n}, b_{n}\right)$ be a disjoint union of intervals. By Theorems 2.5 and 3.6 we have

$$
\begin{gathered}
f\left(b_{n}\right)-f\left(a_{n}\right)=\int_{a_{n}}^{b_{n}} f^{\prime}(t) d t \quad \text { and } \\
\left\langle\Psi\left(b_{n}\right)-\Psi\left(a_{n}\right), f\left(b_{n}\right)\right\rangle=\int_{a_{n}}^{b_{n}}\left\langle\Psi^{\prime}(t), f\left(b_{n}\right)\right\rangle d t,
\end{gathered}
$$

and so, since $f$ and $\Psi$ are continuous, we get

$$
\begin{aligned}
& \sum_{n=1}^{N}\left|z\left(b_{n}\right)-z\left(a_{n}\right)\right| \\
& \quad \leq \sum_{n=1}^{N}\left|\int_{a_{n}}^{b_{n}}\left\langle\Psi^{\prime}(t), f\left(b_{n}\right)\right\rangle d t\right|+\sum_{n=1}^{N}\left|\int_{a_{n}}^{b_{n}}\left\langle\Psi\left(a_{n}\right), f^{\prime}(t)\right\rangle d t\right| \\
& \quad \leq\|f\|_{L^{\infty}(0, T ; X)} \int_{E}\left\|\Psi^{\prime}(t)\right\| d t+\|\Psi\|_{L^{\infty}\left(0, T ; X^{*}\right)} \int_{E}\left\|f^{\prime}(t)\right\| d t .
\end{aligned}
$$

Absolute continuity the Lebesgue integral now implies that $z(t)$ is absolutely continuous, so also $z \in W^{1,1}(0, T)$, with weak derivative $\mathrm{D} z=\frac{d z}{d t}$ almost everywhere. 
Next, Theorem 3.5, (9) and absolute continuity of $z(t)$ imply that the set

$$
\begin{gathered}
E=\left\{t: \frac{d z}{d t} \text { exists, } \quad \lim _{h \rightarrow 0} \frac{1}{h} \int_{0}^{T} f^{\prime}(s) d s=f^{\prime}(t), \quad\right. \text { and } \\
\left.\frac{1}{h} \int_{t}^{t+h}\left\langle\Psi^{\prime}(s) f(s)\right\rangle d s=\left\langle\Psi^{\prime}(t) f(t)\right\rangle\right\}
\end{gathered}
$$

has full measure, $\lambda([0, T] \backslash E)=0$. For each $t \in E$, we have

$$
\begin{aligned}
\frac{d z}{d t}(t) & =\lim _{h \rightarrow 0} \frac{1}{h}(\langle\Psi(t+h)-\Psi(t), f(t)\rangle+\langle\Psi(t), f(t+h)-f(t)\rangle) \\
& =\left\langle\Psi^{\prime}(t), f(t)\right\rangle+\left\langle\Psi(t), f^{\prime}(t)\right\rangle,
\end{aligned}
$$

which is (21). Next, by absolute continuity,

$$
z(T)-z(0)=\int_{0}^{T} \mathrm{D} z(t) d t
$$

which is (22).

\section{Application to Hyperbolic Conservation Laws}

We now apply the calculus we have developed to the Cauchy problem for systems of hyperbolic conservation laws in one space dimension,

$$
\partial_{t} u+\partial_{x} F(u)=0, \quad u(x, 0)=u^{0}(x),
$$

where $u=u(x, t): \mathbb{R} \times \mathbb{R}_{+} \rightarrow \mathbb{R}^{n}, x \in \mathbb{R}$, and $F \in C^{1}\left(\mathbb{R}^{n} ; \mathbb{R}^{n}\right)$.

Recall that a distributional solution of (23) is a measurable function $u(x, t)$ : $\mathbb{R} \times[0, T) \rightarrow \mathbb{R}^{n}$ which satisfies

$$
\begin{aligned}
\int_{0}^{T} \int_{\mathbb{R}}\left(u(x, t)^{\top} \partial_{t} \varphi(x, t)\right. & \left.+F(u(x, t))^{\top} \partial_{x} \varphi(x, t)\right) d x d t \\
& +\int_{R} u^{0}(x)^{\top} \varphi(0, x) d x=0,
\end{aligned}
$$

for every function $\varphi \in C_{c}^{1}\left(\mathbb{R}^{2}, \mathbb{R}^{n}\right)$; here it is implicitly assumed that $F(u(x, t))$ is locally integrable. A distributional solution which is locally bounded is a weak solution.

In defining distributional and weak solutions, there is no distinction between the roles of time and space, with both the solution $u$ and test functions $\varphi$ regarded as functions on $\mathbb{R} \times[0, T)$. Our approach is different, in that we wish to understand the PDE (23) as an evolution equation, so we will regard the solution as a function of time taking values in a Banach space. In particular, we regard the (spatial) test functions as elements of a Banach space $X$, which contains $C_{c}^{\infty}$ as a dense subspace, and, since $u(t)$ acts linearly on these test functions, it has values in $X^{*}$, and we regard $u \in L_{w *}^{q}\left(0, T ; X^{*}\right)$; throughout this section, we fix the constant $1 \leq q \leq \infty$. Taking this point of view, we use the convention that $u(t) \in X^{*}$ stands for $u(\cdot, t)$.

The critical issue is to make sense of the nonlinear flux $F(u)$ and its derivative in the space $X^{*}$. Generally the flux $F$ is given as a function of the conserved variables $u$, regarded as a pointwise field. In our case we treat $u(t) \in X^{*}$ as a field, and we similarly regard $F(u(t)) \in X^{*}$ as a field in the 
same way, being defined via composition. We refer to the corresponding map $F: X^{*} \rightarrow X^{*}$ as a flux mapping. We now assume that $\Omega \subseteq \mathbb{R}$ is open and that the space $X$ of test functions contains $C_{c}^{\infty}(\Omega)$ as a dense subset.

We say that $f \in X^{*}$ has an $X^{*}$-valued distributional derivative, written $\mathbb{D}_{x} f \in X^{*}$, if, for all $\phi \in C_{c}^{\infty}(\Omega) \subset X$, we have

$$
\left|\left\langle f, \phi^{\prime}\right\rangle\right| \leq C\|\phi\|_{X}
$$

and in this case we define $\mathbb{D}_{x} f$ by

$$
\left\langle\mathbb{D}_{x} f, \phi\right\rangle:=-\left\langle f, \phi^{\prime}\right\rangle .
$$

Note that this is not the classical distributional derivative, because we are requiring that $\mathbb{D}_{x} f$ be a bounded operator on $X$.

Definition 4.1. Let $F: X^{*} \rightarrow X^{*}$ be a flux mapping and $u^{0} \in X^{*}$. The function $u \in W_{w *}^{1, q}\left(0, T ; X^{*}\right)$ is called a weak* solution of the system (23) if

$$
u^{\prime}(t)+\mathbb{D}_{x} F(u(t))=0 \text { in } X^{*}
$$

for a.e. $t \in[0, T]$, and such that $\bar{u}(0)=u^{0}$ in $X^{*}$, where $\bar{u}$ is the time continuous representative of $u$. Equivalently, we require

$$
\bar{u}(t)-\bar{u}(s)=\star \int_{s}^{t} \mathbb{D}_{x} F(u(\tau)) d \tau \quad \text { in } X^{*} \quad \text { for } \quad t, s \in[0, T] .
$$

We note that this is a general definition which depends on the choice of the space $X$ of test functions as well as the growth rate $q$.

4.1. Classical Solutions. As a first example, recall that if the system is symmetrizable (say if $F$ is a gradient), then well-known energy estimates yield finite time existence of classical solutions in the space $H^{s}(\mathbb{R})$ for $s>3 / 2[\mathbf{1 3}$. Moreover, since they are differentiable, these satisfy the appropriate integration by parts formulae. Thus, if we choose

$$
X=H^{-s}(\mathbb{R}), \quad \text { so that } \quad X^{*}=H^{s}(\mathbb{R}),
$$

then these classical solutions can be regarded as weak* solutions on $[0, T)$, where $T$ is the blowup time of classical solutions.

To clarify this example, we consider the scalar Burgers' equation,

$$
u_{t}+\left(u^{2} / 2\right)_{x}=0, \quad u(x, 0)=u^{0}(x),
$$

for $u \in \mathbb{R}$, which is well known. Solving by characteristics, we have

$$
u(x, t)=u^{0}\left(x^{0}\right), \quad \text { where } \quad x-x^{0}=u^{0}\left(x^{0}\right) t,
$$

$x^{0}$ being implicitly determined as $x^{0}=x^{0}(x, t)$. Differentiating the equation, we see that the spatial derivative $v(x, t)=u_{x}(x, t)$ satisfies

$$
v_{t}+u v_{x}+v^{2}=0
$$

and solving along characteristics, we find, after simplification,

$$
v(x, t)=\frac{v^{0}\left(x^{0}\right)}{1+t v^{0}\left(x^{0}\right)},
$$

valid as long as $t<-1 / v^{0}\left(x^{0}\right)$; here the blowup time is

$$
t_{b}=\inf \left\{-1 / v^{0}\left(x^{0}\right): v^{0}\left(x^{0}\right)<0\right\} .
$$


Using the quasilinear equation $u_{t}+u u_{x}=0$ directly, we also see that

$$
u^{\prime}(t)=u_{t}(\cdot, t) \quad \text { with } \quad u_{t}(x, t)=\frac{-u^{0}\left(x^{0}\right) v^{0}\left(x^{0}\right)}{1+t v^{0}\left(x^{0}\right)} .
$$

Next, differentiating (27), we get

$$
\frac{\partial x^{0}}{\partial x}=\frac{1}{1+t v^{0}\left(x^{0}\right)} \quad \text { and } \quad \frac{\partial x^{0}}{\partial t}=\frac{-u^{0}\left(x^{0}\right)}{1+t v^{0}\left(x^{0}\right)} .
$$

It follows that the homogeneous $\dot{H}^{1}$ norm of the solution is

$$
\|u(t)\|_{\dot{H}^{1}}^{2}=\int v(x, t)^{2} d x=\int \frac{v^{0}\left(x^{0}\right)^{2}}{\left(1+t v^{0}\left(x^{0}\right)\right)^{3}} d x^{0},
$$

where we have used $d x=\left(1+t v^{0}\left(x^{0}\right)\right) d x^{0}$ from (27) to change variables. Now using (28), we can write

$$
\|u(t)\|_{\dot{H}^{1}}=O(1)\left(1-t / t_{b}\right)^{-3 / 2},
$$

which gives the rate of blowup of $H^{1}$ norm. Thus for any $q>1$, we have $u \in L^{q}\left(0, \tau ; H^{1}\right)$ if and only if $\tau<t_{b}$, the blowup time. Following similar steps, we can calculate higher $H^{s}$ norms and blowup rates, as desired.

This example illustrates that when the class of test functions is large, in this case $H^{-s}$, then the solution is in a smaller space, $H^{s}$, in which we have only local existence. At the other extreme, a very small space such as $H^{s}$ would yield a much larger class of solutions. A more realistic example is obtained by using $X=C_{0}$ as the space of spatial test functions, which would lead to solutions in the space $X^{*}=M$ of time varying Radon measures. This point of view is taken in [14, where the notion of weak* solution allows for vacuums in gas dynamics and fractures in elasticity. These solutions are not weak solutions as vacuums and fractures are represented by delta measures; the flux mapping $F$, as well as the entropy and entropy flux, must be extended to measures (see 14). In general, our goal is to find the right space which would lead to global existence and stability of solutions: in this paper, we primarily focus on weak* solutions $u \in X^{*} \cap B V^{n}$, with $X=C_{0}$ and $X^{*}=M^{n}$.

4.2. BV Weak* Solutions. It is well known that in one space dimension, the correct setting for systems of hyperbolic conservation laws is the space $B V$ of functions of bounded variation $\left[\mathbf{9}, \mathbf{2}, \mathbf{5}\right.$. Recall that if $w \in B V_{l o c}$ then $\mathbb{D} w \in M_{l o c}$, which is the dual of $C_{0}$. We will thus take

$$
X=C_{0}(\mathbb{R})^{n}, \quad \text { so that } X^{*}=M_{l o c}(\mathbb{R})^{n},
$$

and use $M_{l o c}$ to define weak* solutions. However, we wish to restrict our solutions further so that the ODE remains consistent. We accomplish this as follows: if $\Phi \in W_{w *}^{1, q}\left(0, T ; X^{*}\right)$, and in addition, $\Phi$ has values in some $Y \subset X^{*}$, then we write $\Phi \in W_{w *}^{1, q}\left(0, T ; Y, X^{*}\right)$, that is we set

$$
W_{w *}^{1, q}\left(0, T ; Y, X^{*}\right)=\left\{\Phi \in W_{w *}^{1, q}\left(0, T ; X^{*}\right): \Phi(t) \in Y, t \in[0, T]\right\} .
$$

Note that we do not assume that $Y$ is a subspace of $X^{*}$, because we use the topology of $X^{*}$ throughout. We also use the notation $T^{-}$to mean up to but not including $T$, so that

$$
L_{w *}^{q}\left(0, T^{-} ; X^{*}\right):=\left\{\phi:[0, T) \rightarrow X^{*}: \phi \in L_{w *}^{q}\left(0, \tau ; X^{*}\right) \forall 0<\tau<T\right\},
$$


and similarly for $W_{w *}^{1, q}\left(0, T^{-} ; X^{*}\right)$.

For one-dimensional systems of conservation laws, for which $B V$ solutions are appropriate, we take $X=\left(C_{0}\right)^{n}, X^{*}=M_{l o c}^{n}$, and $Y=B V_{l o c}^{n}$.

Definition 4.2. Suppose that $u^{0} \in M_{l o c}\left(\mathbb{R} ; \mathbb{R}^{n}\right)$. The function

$$
u \in W_{w *}^{1, q}\left(0, T^{-} ; B V_{l o c}^{n}, M_{l o c}^{n}\right)
$$

is called a $\mathrm{BV}$ weak* solution to the Cauchy problem (23) if for a.e. $t \in(0, T)$

$$
u^{\prime}(t)+\mathbb{D}_{x} F(u(t))=0 \quad \text { in } \quad M_{l o c}^{n}
$$

and such that $\bar{u}(0)=u^{0}$, where $\bar{u}$ is the time continuous representative of $u$ in $M_{l o c}^{n}$.

In (29), $u^{\prime}$ is the G-weak derivative of $u$ in the space $W_{w *}^{1, q}\left(0, T^{-} ; M_{l o c}^{n}\right)$, and $\mathbb{D}_{x} F(u(t))$ is the distributional derivative of the function $x \rightarrow F(u(x, t))$. Since $u(t) \in B V_{l o c}^{n}$ for a.e. $t$, and $F$ is $C^{1}$ and so Lipschitz, for these $t$ we also have $F(u(\cdot, t)) \in B V_{l o c}^{n}$, and Lemma 2.1 implies that $\mathbb{D}_{x} F(u(t)) \in M_{l o c}^{n}$, so we can understand the equation in $M_{l o c}^{n}$. Moreover, in view of separability of $C_{0}$, each element of $L_{w *}^{q}\left(0, T^{-} ; M_{l o c}^{n}\right)$ is norm-measurable, and (29) is equivalent to requiring that $\left[u^{\prime}\right]+\left[\mathrm{D}_{x} F(u)\right]=0$ as equivalence classes of $L_{w *}^{q}\left(0, T ; M^{n}(\Omega)\right)$. As usual, this means

$$
\bar{u}(t)-\bar{u}(s)=\star \int_{s}^{t} \mathbb{D}_{x} F(u(\tau)) d \tau \in M_{l o c} \quad \text { for } \quad t, s \in[0, T] .
$$

We note that our definition of weak* solutions implicitly requires some regularity, namely that $u$ is absolutely continuous as a function $t \rightarrow u(t) \in M_{l o c}^{n}$ with values in $B V_{l o c}^{n}$. Also, recall that we require only that $u^{\prime}$ (and thus $\mathbb{D}_{x} F(u)$ ) be weak*-measurable, rather than strongly measurable, and the parameter $q$ allows a range of growth rates of weak* solutions. In this sense, our weak* solutions are different from weak solutions, which are locally bounded locally integrable functions $u(x, t)$ satisfying (24). Although the two notions of solution are different, we show they yield the same solutions in the most important case.

Theorem 4.3. Suppose that $u \in W_{w *}^{1, q}\left(0, T^{-} ; B V_{l o c}^{n}, M_{l o c}^{n}\right)$ is a $B V$ weak ${ }^{*}$ solution to the Cauchy problem (23), with continuous representative $\bar{u}$. Then $\bar{u}$ is Hölder continuous as a function into $L_{\text {loc }}^{1}\left(\mathbb{R} ; \mathbb{R}^{n}\right)$, that is,

$$
\bar{u} \in C^{0,1-1 / q}\left(0, T^{-} ; L_{l o c}^{1}\right) \quad \text { or } \quad \bar{u} \in \operatorname{Lip}\left(0, T^{-} ; L_{l o c}^{1}\right) \text {, }
$$

for $1 \leq q<\infty$ or $q=\infty$, respectively. The function $\bar{u}(x, t)$ is a distributional solution of the Cauchy problem (23). In particular, if $u$ is locally bounded, $u \in L_{w *}^{\infty}\left(0, T^{-} ; L_{l o c}^{\infty}\left(\mathbb{R} ; \mathbb{R}^{n}\right)\right)$, then $\bar{u}(x, t)$ is a weak solution to the Cauchy problem (23).

Proof. According to Theorem A.2 below, $\bar{u} \in \widetilde{W}^{1, q}\left(0, T^{-} ; M_{l o c}^{n}\right)$, which implies that for each open $\Omega \subset \subset \mathbb{R}$, there exists $\phi \in L^{q}$ such that

$$
\|\bar{u}(t)-\bar{u}(s)\|_{M^{n}(\Omega)} \leq \int_{s}^{t} \phi(\tau) d \tau, \quad t, s \in[0, \tau] .
$$

Since $\phi \in L^{q}$, we apply Hölder's inequality, together with (5), to get

$$
\|\bar{u}(t)-\bar{u}(s)\|_{L^{1}(\Omega)}=\|\bar{u}(t)-\bar{u}(s)\|_{M^{n}(\Omega)} \leq\|\phi\|_{q}|t-s|^{1-1 / q},
$$


which implies (30).

To show that $\bar{u}$ is a distributional solution, assume we are given a test function $\psi \in C_{c}^{1}\left(\mathbb{R} \times(-\infty, T) ; \mathbb{R}^{n}\right)$. Restricting $\psi$ to the time interval $[0, T)$, we have $\psi \in W^{1, \infty}\left(0, \tau ; C_{0}\left((a, b) ; \mathbb{R}^{n}\right)\right)$ for some $0<\tau<T$ and finite open interval $(a, b) \in \mathbb{R}$. From (29) it follows that $u^{\prime}=-\mathbb{D}_{x} F(u) \in L_{w *}^{1}\left(0, \tau ; M^{n}(a, b)\right)$, and by Theorem 3.8, the functions $t \rightarrow\left\langle\mathbb{D}_{x} F(u(t)), \psi(t)\right\rangle$ and $t \rightarrow\langle u(t), \psi(t)\rangle$ are Lebesgue integrable and

$$
\begin{aligned}
\int_{0}^{\tau}\left\langle\mathbb{D}_{x} F(u(t)), \psi(t)\right\rangle d t & =-\int_{0}^{\tau}\left\langle u^{\prime}(t), \psi(t)\right\rangle d t \\
& =\langle\bar{u}(0), \psi(0)\rangle+\int_{0}^{\tau}\left\langle u(t), \psi^{\prime}(t)\right\rangle d t
\end{aligned}
$$

Next, for each $t \in[0, T)$ we have $u(t) \in B V_{l o c}^{n}$, and since $F$ is $C^{1}$, we have also $F(u(t)) \in B V_{l o c}^{n}$, so that $\mathbb{D}_{x} F(u(t)) \in M_{l o c}^{n}$ and

$$
\begin{aligned}
\left\langle\mathbb{D}_{x} F(u(t)), \psi(t)\right\rangle & =\int_{\mathbb{R}} \psi(x, t) \mathbb{D}_{x}(F(u))(d x, t) \\
& =-\int_{\mathbb{R}} F(u(x, t))^{\top} \partial_{x} \psi(x, t) d x .
\end{aligned}
$$

Similarly, since $u(t) \in M_{l o c}^{n} \bigcap B V_{l o c}^{n} \subset L_{l o c}^{1}\left(\mathbb{R} ; \mathbb{R}^{n}\right)$,

$$
\begin{aligned}
& \left\langle u(t), \psi^{\prime}(t)\right\rangle=\int_{\mathbb{R}} \partial_{t} \psi(x, t) \cdot u(d x, t)=\int_{\mathbb{R}} u(x, t)^{\top} \partial_{t} \psi(x, t) d x, \text { and } \\
& \langle\bar{u}(0), \psi(0)\rangle=\int_{\mathbb{R}} \psi(x, 0) \cdot \bar{u}(d x, 0)=\int_{\mathbb{R}} \bar{u}(x, 0)^{\top} \psi(x, 0) d x .
\end{aligned}
$$

Combining the above relations we obtain

$$
\begin{aligned}
\int_{0}^{T} \int_{\mathbb{R}}\left(u(x, t)^{\top} \partial_{t} \psi(x, t)\right. & \left.+F(u(x, t))^{\top} \partial_{x} \psi(x, t)\right) d x d t \\
& +\int_{\mathbb{R}} u^{0}(x)^{\top} \psi(x, 0) d x=0,
\end{aligned}
$$

which is (24). Finally, since $\bar{u}(t)$, and hence also $F(\bar{u}(t))$, is continuous with values in $L_{l o c}^{1}$, both $\bar{u}(x, t)$ and $F(\bar{u})(x, t)$ are locally integrable, and the proof is complete.

Having shown that any weak* solution is a distributional solution, we now show that a weak solution with sufficiently regular growth is also a weak* solution. In particular, the global weak solutions generated by Glimm's method, front tracking, and vanishing viscosity, all of which have uniformly bounded total variation, are all weak ${ }^{*}$ solutions. It follows that the uniqueness and $L^{1}$ stability results of Bressan et.al. hold unchanged in the framework of weak* solutions.

Theorem 4.4. Let $u(x, t)$ be a weak solution of the Cauchy problem (23), with $u(\cdot, t) \in B V_{\text {loc }}^{n}$ for each $t \in[0, T)$. Suppose also that for each open interval $\Omega \subset \subset \mathbb{R}$, there is some $g_{\Omega} \in L^{q}\left(0, T^{-}\right)$such that

$$
\mathbb{V}(u(\cdot, t) ; \Omega) \leq g_{\Omega}(t), \quad \text { a.e. } t \in(0, T) .
$$

Then $u \in W_{w *}^{1, q}\left(0, T^{-} ; B V_{l o c}^{n}, M_{l o c}^{n}\right)$, and $u$ is a $B V$ weak* solution to the Cauchy problem. 
Proof. Let $I=(a, b) \subset \subset(0, T)$ and $\Omega \subset \mathbb{R}$ be finite intervals. Since $u(x, t)$ is locally bounded and integrable, for each $\alpha \in C_{0}^{1}(\Omega)$, the map $t \rightarrow\langle u(t), \alpha\rangle:=$ $\int_{\Omega} u(x, t) \alpha(x) d x$ is measurable and integrable over $I$. Also,

$$
\underset{t \in I}{\operatorname{ess} \sup }\|u(\cdot, t)\|_{M^{n}(\Omega)}=\underset{t \in I}{\operatorname{esssup}} \int_{\Omega}|u(x, t)| d x \leq \lambda(\Omega)\|u\|_{L^{\infty}(I \times \Omega)},
$$

so that $u \in L_{w *}^{\infty}(a, b ; M(\Omega))$.

Next, for each $t$, we have $u(t):=u(\cdot, t) \in B V_{l o c}^{n}$, and so since $F \in C^{1}$, also $F(u(t)) \in B V_{l o c}^{n}$, and in particular, $x \rightarrow F(u(x, t))$ is integrable over $\Omega$ for each $t \in I$. Lemma 2.1 now implies that $\mathbb{D}_{x} F(u(t)) \in M_{\text {loc }}^{n}$ for each $t \in I$, and

$$
t \rightarrow\left\langle\mathbb{D}_{x} F(u(t)), \alpha\right\rangle=-\int_{\mathbb{R}} F(u(x, t)) \alpha^{\prime}(x) d x
$$

is measurable and integrable on $I$. Moreover, since $F$ is locally Lipschitz and $u$ is locally bounded, our assumption (31) implies that there is some $\widetilde{g}_{\Omega} \in$ $L^{q}\left(0, T^{-}\right)$such that

$$
\left\|\mathbb{D}_{x} F(u(t))\right\|_{M^{n}(\Omega)}=\mathbb{V}(F(u(\cdot, t)) ; \Omega) \leq \widetilde{g}_{\Omega}(t), \quad \text { a.e. } t \in(0, T),
$$

and so also

$$
\left|\left\langle\mathbb{D}_{x} F(u(t)), \alpha\right\rangle\right| \leq\left\|\mathbb{D}_{x} F(u(t))\right\|_{M^{n}(\Omega)}\|\alpha\|_{L^{\infty}(\Omega)} \leq \widetilde{g}_{\Omega}(t)\|\alpha\|_{L^{\infty}(\Omega)} .
$$

Since $\alpha$ is arbitrary and $g_{\Omega} \in L^{q}\left(0, T^{-}\right)$, Theorem A.1 below implies that

$$
\mathbb{D}_{x} F(u(t)) \in L_{w *}^{q}\left(0, T^{-} ; M^{n}(\Omega)\right) .
$$

Now let $\psi \in C_{c}^{1}(I)$ and $\alpha \in C_{0}^{1}(\Omega)$, and set $\varphi(x, t)=\psi(t) \alpha(x)$. Using (24), we get

$$
\int_{I}\left(\int_{\Omega} u(x, t)^{\top} \alpha(x) d x\right) \psi^{\prime}(t) d t+\int_{I}\left(\int_{\Omega} F(u(x, t))^{\top} \alpha^{\prime}(x) d x\right) \psi(t) d t=0,
$$

or equivalently

$$
\int_{I}\langle u(t), \alpha\rangle \psi^{\prime}(t) d t=\int_{I}\left\langle\mathbb{D}_{x} F(u(t)), \alpha\right\rangle \psi(t) d t .
$$

Since $\psi$ is arbitrary, it follows that the G-weak derivative $u^{\prime}$ exists and

$$
\left\langle u^{\prime}(t), \alpha\right\rangle=-\left\langle\mathbb{D}_{x} F(u(t)), \alpha\right\rangle \quad \text { a.e. } t \in I .
$$

Since $\alpha$ and $I=(a, b) \subset \subset(0, T)$ are arbitrary we conclude that

$$
u^{\prime}+\mathbb{D}_{x}(F(u(t)))=0 \quad \text { in } M^{n}(\Omega), \text { a.e. } t \in(0, T)
$$

and $u \in W_{w *}^{1, q}\left(0, T^{-} ; M^{n}(\Omega)\right)$. Moreover, (31) and Theorem A.1 below yield $u \in L_{w *}^{q}\left(0, T^{-} ; B V^{n}(\Omega)\right)$, so also $u \in W_{w *}^{1, q}\left(0, T^{-} ; B V^{n}(\Omega), M^{n}(\Omega)\right)$, and $u$ is a BV weak* solution.

It remains to show that the initial data is taken on in an appropriate sense. Let $\bar{u}(t)$ be the continuous representative of the weak solution $u(\cdot, t) \in M^{n}(\Omega)$. We have shown that this is a weak* solution, with initial data $\bar{u}(0) \in B V_{l o c}^{n}$. By Theorem 4.3, this is a continuous distributional solution. Moreover, $u(t)=\bar{u}(t)$ a.e. $t$ in $M^{n}(\Omega)$, and so also in $L_{l o c}^{1}(\Omega)$, and this in turn implies $u(x, t)=\bar{u}(x, t)$ 
almost surely as functions of both space and time. Since both $u$ and $\bar{u}$ are distributional solutions, for any $\varphi \in C_{c}^{1}(\Omega \times(-\infty, \tau))$, use of (24) then yields

$$
\int_{\mathbb{R}} \bar{u}(x, 0)^{\top} \varphi(0, x) d x=\int_{\mathbb{R}} u^{0}(x)^{\top} \varphi(0, x) d x,
$$

so that $\bar{u}(0, x)=u^{0}(x)$ a.e. $x$, and thus $\bar{u}(0)=u^{0} \in M_{l o c}^{n}$.

4.3. Shock Waves and Entropy. It is well known that discontinuities in weak solutions satisfy the Rankine-Hugoniot jump conditions, and that entropy conditions are required to select the unique, physically relevant solution when discontinuities are present. Here we restate these conditions from the point of view of weak* solutions. In particular, we note the ease with which the Rankine-Hugoniot conditions are derived.

In deriving the shock conditions, we wish to understand the local pointwise structure of BV weak* solutions. To do so, we make some reasonable simplifying assumptions. For a given $\Omega \subset \mathbb{R}$ and $t \in[0, T)$, we assume $u(t) \in S B V(\Omega)$, so that $u$ has no singular continuous part, and we can write

$$
u(x, t)=\sum_{j} v_{j}(t) H\left(x-x_{j}(t)\right)+u_{c}(x, t), \quad \text { a.e. } x \in \Omega,
$$

where $u_{c}$ is the absolutely continuous part of $u$, and there are jumps of size $v_{j}(t)$ located at points $x_{j}(t) \in \mathcal{J} \subset \Omega$. The jump $v_{j}(t)$ is

$$
v_{j}(t)=u\left(x_{j}(t)+, t\right)-u\left(x_{j}(t)-, t\right),
$$

while $u_{c}(\cdot, t) \in W^{1,1}(\mathbb{R})$ with $\partial_{x} u=\partial_{x} u_{c}$ almost surely, where we have used $\partial_{x}$ to denote the pointwise (partial) derivative. It follows that $F(u)$ has the same form, namely

$$
F(u(x, t))=\sum_{j} g_{j}(t) H\left(x-x_{j}(t)\right)+f_{c}(x, t), \quad \text { a.e. } x \in \Omega,
$$

where $g_{j}(t)$ is the jump in $F(u)$ at $x_{j}(t)$,

$$
g_{j}(t)=F\left(u\left(x_{j}(t)+, t\right)\right)-F\left(u\left(x_{j}(t)-, t\right)\right),
$$

and $f_{c}$ is given by

$$
f_{c}(x, t)=F\left(u_{c}(x, t)+\sum_{x_{j} \leq x} v_{j}(t)\right)-\sum_{x_{j} \leq x} g_{j}(t),
$$

so also $f_{c}(\cdot, t) \in W^{1,1}(\mathbb{R})$. From (33), we calculate the distributional derivative

$$
\mathbb{D}_{x} F(u)=\sum_{j} g_{j}(t) \delta_{x_{j}(t)}+\partial_{x} f_{c}(\cdot, t) \in M^{n}(\Omega),
$$

where $\partial_{x} f_{c}(\cdot, t)$ is defined $\lambda$-a.e., and in fact, for almost all $x \neq x_{j}(t)$,

$$
\partial_{x} f_{c}(x, t)=D F(u) \partial_{x} u_{c}(x, t)=D F(u) \partial_{x} u(x, t) \in L^{1}(\mathbb{R}),
$$

where $D F(u)$ is the derivative of $F: \mathbb{R}^{n} \rightarrow \mathbb{R}^{n}$.

We now make the further assumption that $u_{c}(x, \cdot), v_{j}$ and $x_{j}$ are absolutely continuous functions of $t$, a.e $x \in \Omega$. Thus for a full measure set of $t$, say $t \in E$, 
the weak derivative $\mathbb{D}_{t} u$ can be calculated as a measure, and we have

$$
\begin{aligned}
\mathbb{D}_{t} u=\sum_{j} v_{j}(t) \frac{d\left(-x_{j}\right)}{d t} \delta_{x_{j}(t)} \\
\quad+\sum_{j} \frac{d v_{j}}{d t} H\left(x-x_{j}(t)\right)+\partial_{t} u_{c}(x, t) \in M^{n}(\Omega) .
\end{aligned}
$$

Since $u$ is assumed to be a weak* solution, it follows that its G-weak derivative $u^{\prime} \in M^{n}(\Omega)$, and $u^{\prime}=\mathbb{D}_{t} u$ whenever $\mathbb{D}_{t} u$ exists as a measure, and therefore that for $t \in E$,

$$
\mathbb{D}_{t} u+\mathbb{D}_{x} F(u)=0 \in M^{n}(\Omega) .
$$

For two measures to be equal their atomic parts must coincide, and comparing (34) and (35) yields $v_{j}(t) \frac{d x_{j}}{d t}=g_{j}(t)$, which is the Rankine-Hugoniot condition,

$$
F\left(u\left(x_{j}(t)+, t\right)\right)-F\left(u\left(x_{j}(t)-, t\right)\right)=x_{j}^{\prime}(t)\left(u\left(x_{j}(t)+, t\right)-u\left(x_{j}(t)-, t\right)\right),
$$

for each $j \in \mathcal{J}$. Moreover, away from the jump set, the absolutely continuous parts of the measures must agree a.e., so that for a.e. $x \neq x_{j}(t)$,

$$
\sum_{x_{j}<x} \frac{d v_{j}}{d t}+\partial_{t} u_{c}(x, t)+\partial_{x} f_{c}(x, t)=0,
$$

and using (32), this yields

$$
\partial_{t} u(x, t)+D F(u) \partial_{x} u(x, t)=0, \quad \text { a.e. } x \neq x_{j},
$$

so that, as is well known, the absolutely continuous part of the solution satisfies the quasilinear form of the equation almost everywhere. Note that the jump conditions (36) are trivially satisfied at any point of continuity of $u$.

Having checked that discontinuities in weak* solutions satisfy the RankineHugoniot condition (36), we must now give an entropy selection criterion which will ensure uniqueness of solutions with shocks. For abstract systems that are genuinely nonlinear and/or linearly degenerate, there are two such conditions, namely the Lax entropy condition and that obtained from a convex entropy/flux pair. Here we give the appropriate statement of these entropy conditions for weak* solutions.

Recall that the Lax entropy condition holds for a single isolated jump associated with a specific wave family. We can rewrite (36) for a single isolated jump located at $x=\xi(t)$ as

$$
F\left(u_{+}\right)-F\left(u_{-}\right)=\xi^{\prime}(t)\left(u_{+}-u_{-}\right), \quad \text { where } u_{ \pm}=u(\xi(t) \pm, t),
$$

which is an eigenvalue problem, with $n$ different solutions, one for each wave family. The wave families are in turn distinguished by the associated nonlinear wave speed $\lambda_{k}$, the $k$-th eigenvalue of the matrix $D F(u)$. Lax's entropy criterion for a $k$-shock is then usually written as

$$
\lambda_{k}\left(u_{-}\right)>\xi^{\prime}(t)>\lambda_{k}\left(u_{+}\right),
$$

so the associated $k$-th characteristics impinge on both sides of the shock. We continue to use this condition for isolated shocks in weak* solutions, for which the left and right limits are well-defined functions. 
On the other hand, if we are given a $C^{1}$ entropy/flux pair $(\eta, q)$, we require that the map

$$
t \mapsto \eta(u(\cdot, t)) \in W_{w *}^{1,1}\left(0, T ; M_{l o c}\right),
$$

and the usual entropy condition holds, namely

$$
\mu_{\eta}:=\eta(u)^{\prime}+\mathbb{D}_{x} q(u) \leq 0 \quad \text { in } M .
$$

This can be interpreted as a measure, since every signed distribution is representable as a signed measure. Note that since $\eta$ and $q$ are $C^{1}$ functions, use of (37) at points of absolute continuity of $u(x, t)$ means that the measure $\mu_{\eta}$ vanishes at those points, so that $\mu_{\eta}$ is supported on the jump set of the solution. We note that the requirement that $\eta \in W_{w *}^{1,1}\left(0, T ; M_{l o c}\right)$ is stronger than the usual assumption that the sum $\eta_{t}+q_{x}=\operatorname{div}(\eta, q) \leq 0$ in $M$, because we require each term to be a measure separately.

4.4. The Riemann Problem. As an illustrative example, we describe the well-known solution of the Riemann problem as a $B V$ weak* solution. Recall that the solution of the Riemann problem consists of constant states separated by $n$ centered elementary waves, these being (centered) shocks or rarefactions for genuinely nonlinear families, and (centered) contact discontinuities for linearly degenerate families.

Recall that a centered $k$-shock consists of a discontinuity between two constant states $u_{-}=u_{k-1}$ and $u_{+}=u_{k}$ satisfying (38) and (39), which has constant shock speed $\xi^{\prime}(t)=\sigma_{k}\left(u_{k-1}, u_{k}\right)$, so that the position of the shock at time $t$ is given by $\xi(t)=\sigma_{k}\left(u_{k-1}, u_{k}\right) t$. The centered $k$-shock can thus be written as

$$
u(x, t)=u_{k-1}+\left(u_{k}-u_{k-1}\right) H\left(x-\sigma_{k}\left(u_{k-1}, u_{k}\right) t\right) .
$$

A centered rarefaction wave is a solution of the form $u(x, t)=w(x / t)$, which satisfies (37), which reduces to

$$
-\varepsilon \frac{d w}{d \varepsilon}+D F(w(\varepsilon)) \frac{d w}{d \varepsilon}=0, \quad \varepsilon=x / t .
$$

This is an eigenvalue problem, and the centered $k$-rarefaction wave is $u(x, t)=$ $w_{k}(\varepsilon)$, corresponding to the $k$-th eigenpair,

$$
\frac{d w_{k}}{d \varepsilon}=r_{k}(\varepsilon) \quad \text { and } \quad \varepsilon=\frac{x}{t}=\lambda_{k}\left(w_{k}(\varepsilon)\right),
$$

and such that $\lambda_{k}\left(w_{k}(\varepsilon)\right)$ is monotone increasing. The $k$-rarefaction between states $u_{k-1}$ and $u_{k}$ is then described by

$$
u(x, t)= \begin{cases}u_{k-1}, & x / t \leq \lambda_{k}\left(u_{k-1}\right), \\ w_{k}(\varepsilon), & \lambda_{k}\left(u_{k-1}\right) \leq x / t=\varepsilon \leq \lambda_{k}\left(u_{k}\right), \\ u_{k}, & \lambda_{k}\left(u_{k}\right) \leq x / t .\end{cases}
$$

If the $k$-th family is linearly degenerate, that is $r_{k} \cdot \nabla \lambda_{k} \equiv 0$, then the wave speed does not change across a $k$-wave, and both (41) and (43) degenerate and coincide, and the $k$-contact discontinuity is given by

$$
\begin{aligned}
u(x, t) & =u_{k-1}+\left(u_{k}-u_{k-1}\right) H\left(x-\lambda_{k}\left(u_{k}\right) t\right), \\
\lambda_{k}\left(u_{k}\right) & =\lambda_{k}\left(u_{k-1}\right) .
\end{aligned}
$$


The general Riemann problem with data $u^{0}(x)=u_{L}+\left(u_{R}-u_{L}\right) H(x)$ is solved by finding (unique) states $\left\{u_{\ell}\right\}_{\ell=0}^{n}$, with $u^{0}=u_{L}$ and $u_{n}=u_{R}$, such that each $u_{k-1}$ is connected to $u_{k}$ by a centered $k$-wave. We describe this as a weak* solution by explicitly calculating the appropriate derivatives. It suffices to consider the waves separately.

We first consider a $k$-shock satisfying (41). Clearly

$$
F(u(x, t))=F\left(u_{k-1}\right)+\left(F\left(u_{k}\right)-F\left(u_{k-1}\right)\right) H\left(x-\sigma_{k}\left(u_{k-1}, u_{k}\right) t\right),
$$

and differentiating yields

$$
\begin{aligned}
u^{\prime} & =\mathbb{D}_{t} u=-\left(u_{k}-u_{k-1}\right) \sigma_{k} \delta_{\sigma_{k} t} \quad \text { and } \\
\mathbb{D}_{x} F(u) & =\left(F\left(u_{k}\right)-F\left(u_{k-1}\right)\right) \delta_{\sigma_{k} t},
\end{aligned}
$$

where $u^{\prime}$, being strongly measurable in $M_{l o c}^{n}$, is the B-weak derivative of $u$. The jump condition (38) implies (29), and $u^{\prime}$ is clearly bounded,

$$
\begin{aligned}
\left|\left\langle u^{\prime}, \alpha\right\rangle\right| & =\left|u_{k}-u_{k-1}\right|\left|\sigma_{k}\right|\|\alpha\|, \quad \text { and } \\
\left\|u^{\prime}\right\|_{M^{n}} & =\left|u_{k}-u_{k-1}\right|\left|\sigma_{k}\right|,
\end{aligned}
$$

so $u \in W^{1, \infty}\left(0, \infty^{-} ; B V_{l o c}^{n}, M_{l o c}^{n}\right)$. The same estimates hold for $k$-contacts.

We now describe the $k$-rarefaction similarly. Across the wave, namely for $\lambda_{k}\left(u_{k-1}\right) \leq x / t \leq \lambda_{k}\left(u_{k}\right)$, we have

$$
u^{\prime}=\frac{d w_{k}}{d \varepsilon} \partial_{t} \varepsilon \quad \text { and } \quad \mathbb{D}_{x} F(u)=D F \frac{d w_{k}}{d \varepsilon} \partial_{x} \varepsilon,
$$

where $x=\lambda_{k}\left(w_{k}(\varepsilon)\right) t$, and $u^{\prime}=\mathbb{D}_{x} F(u)=0$ otherwise. It follows that

$$
1=\dot{\lambda}_{k} t \partial_{x} \varepsilon \quad \text { and } \quad 0=\lambda_{k}+\dot{\lambda}_{k} t \partial_{t} \varepsilon,
$$

where $\dot{\lambda}_{k}:=\frac{d \lambda_{k}}{d \varepsilon}=\frac{d w_{k}}{d \varepsilon} \cdot \nabla \lambda_{k}$, and we can calculate the action of $u^{\prime}$ on a test function $\alpha$, by

$$
\left\langle u^{\prime}, \alpha\right\rangle=\int_{t \lambda_{k}\left(u_{k-1}\right)}^{t \lambda_{k}\left(u_{k}\right)} \frac{d w_{k}}{d \varepsilon} \frac{-\lambda_{k}}{t \dot{\lambda}_{k}} \alpha(x) d x=-\int_{\lambda_{k}\left(u_{k-1}\right)}^{\lambda_{k}\left(u_{k}\right)} \frac{d F}{d \lambda} \alpha(\lambda t) d \lambda,
$$

by change of variables, where now $\lambda$ parameterizes the integral curve. It follows that $u^{\prime}$ is bounded,

$$
\left|\left\langle u^{\prime}, \alpha\right\rangle\right| \leq \int_{\lambda_{k}\left(u_{k-1}\right)}^{\lambda_{k}\left(u_{k}\right)}\left|\frac{d F}{d \lambda}\right| d \lambda\|\alpha\|, \quad \text { so that } \quad\left\|u^{\prime}\right\|_{M^{n}} \leq \mathbb{V}(F),
$$

the variation being taken along the integral curve, and again the solution satisfies $u \in W^{1, \infty}\left(0, \infty^{-} ; B V_{l o c}^{n}, M_{l o c}^{n}\right)$.

Having described the simple waves separately, we now combine them into Lax's well-known solution of the general Riemann problem. That is, we identify states $u_{0}=u_{L}, u_{1}, \ldots, u_{n}=u_{R}$, such that each pair $\left(u_{k-1}, u_{k}\right)$ is joined by a $k$-wave, satisfying (41) or (43) for genuinely nonlinear fields, and (44) for linearly degenerate fields. Our assumption of strict hyperbolicity means that for each $k=1, \ldots, n$, we have $\lambda_{k}\left(u_{k}\right)<\lambda_{k+1}\left(u_{k}\right)$, so the waves can be consistently pieced together for positive times $t>0$. This means that the calculations above all hold locally, and for $t>0$, the (Bochner) derivative $u^{\prime}$ is simply the sum of each of the individual terms.

It remains to state the sense on which the initial data is taken on. Recall that the solution is absolutely continuous in $M^{n}$, and as $t \rightarrow 0$, each individual 
wave converges in $M^{n}$ to the limit $u_{k-1}+\left(u_{k}-u_{k-1}\right) H(x)$, so the full solution converges to $u_{L}+\left(u_{R}-u_{L}\right) H(x)$, as required. Here, since for each fixed $t$, $u(\cdot, t) \in B V \subset L_{l o c}^{1} \subset M_{l o c}^{n}$, we note that $\|u(\cdot, t)\|_{M^{n}}=\|u(\cdot, t)\|_{L^{1}}$, so we have continuity in $L^{1}$ as a function of $t$, namely $u(\cdot, t) \rightarrow u^{0} \in L^{1}$. We note that generally, the solution is not continuous at $t=0$ in $B V$, but only in the larger space $L^{1}$. However, the Riemann solution is continuous as a function of $t$ in $B V$ (with constant $B V$ norm) for all positive times $t>0$.

\section{Appendix A. G-weak Differentiability}

We now show that if a function $\Psi \in L_{w *}^{q}\left(0, T ; X^{*}\right)$ is bounded by a sufficiently regular integral, then it is G-weakly differentiable. Throughout this appendix, we assume $p$ and $q$ satisfy $\frac{1}{p}+\frac{1}{q}=1$.

Theorem A.1. Fix $\psi_{0} \in X^{*}$ and suppose that $\Psi \in L_{w *}^{q}\left(0, T ; X^{*}\right)$ is such that for each $x \in X$, there are functions $v_{x} \in L^{1}(0, T)$ such that

$$
\langle\Psi(t), x\rangle=\left\langle\psi_{0}, x\right\rangle+\int_{0}^{t} v_{x}(s) d s, \quad \text { a.e. } t \in[0, T],
$$

and suppose there is a non-negative $v \in L^{q}(0, T)$ such that

$$
\left|v_{x}(t)\right| \leq v(t)\|x\|, \quad \text { a.e. } t \in[0, T] .
$$

Then we have $[\Psi] \in W_{w *}^{1, q}\left(0, T ; X^{*}\right)$ if either $1<q \leq \infty$ or $q=1$ and $X$ is separable.

Proof. Recalling that $L^{p}(0, T ; X)^{*} \simeq L_{w *}^{q}\left(0, T ; X^{*}\right)$, we construct a bounded linear functional on the space $L^{p}(0, T ; X)$ which is a G-weak derivative of $[\Psi]$.

We begin by observing that $v_{x}$ is almost linear in $x$; that is, for fixed $x_{1}$, $x_{2} \in X$ and scalars $\alpha_{1}, \alpha_{2}$, we have

$$
\int_{0}^{t} v_{\alpha_{1} x_{1}+\alpha_{2} x_{2}}(s) d s=\int_{0}^{t} \alpha_{1} v_{x_{1}}(s)+\alpha_{2} v_{x_{2}}(s) d s, \quad \text { a.e. } t,
$$

and so absolute continuity of the integral implies that, for a.e. $t$, we have

$$
v_{\alpha_{1} x_{1}+\alpha_{2} x_{2}}(t)=\alpha_{1} v_{x_{1}}(t)+\alpha_{2} v_{x_{2}}(t) .
$$

We first assume $q>1$ and let $h(t)=\sum_{n=1}^{N} x_{n} \mathcal{X}_{E_{n}}(t) \in X$ be a simple function. Define the functional $\Gamma$ on simple functions by

$$
\langle\Gamma, h\rangle:=\sum_{n=1}^{N} \int_{E_{n}} v_{x_{n}}(t) d t .
$$

It is easy to verify that $\langle\Gamma, h\rangle$ is independent of the representation of $h$, and

$$
\left\langle\Gamma, \alpha_{1} h_{1}+\alpha_{2} h_{2}\right\rangle=\alpha_{1}\left\langle\Gamma, h_{1}\right\rangle+\alpha_{2}\left\langle\Gamma, h_{2}\right\rangle,
$$

so that $\Gamma$ is linear on the subspace of simple functions.

Recall that for simple $h(t)$ we can take the $E_{n}$ disjoint, in which case

$$
\|h\|_{L^{p}(0, T ; X)}=\left(\int_{0}^{T}\left\|\sum_{n} x_{n} \mathcal{X}_{E_{n}}(t)\right\|^{p} d t\right)^{1 / p}=\left(\sum_{n}\left\|x_{n}\right\|^{p} \lambda\left(E_{n}\right)\right)^{1 / p},
$$


and for such $h$, we have

$$
\begin{aligned}
|\langle\Gamma, h\rangle| & \leq \sum_{n=1}^{N}\left\|x_{n}\right\| \int_{E_{n}} v(t) d t=\int_{0}^{T} v(t)\left(\sum_{n=1}^{N}\left\|x_{n}\right\| \mathcal{X}_{E_{n}}(t)\right) d t \\
& \leq\|v\|_{L^{q}(0, T)}\left(\sum_{i=1}^{N}\left\|x_{n}\right\|^{p} \lambda\left(E_{n}\right) d t\right)^{1 / p}=\|v\|_{L^{q}(0, T)}\|h\|_{L^{p}(0, T ; X)},
\end{aligned}
$$

where we have used (46) and Young's inequality.

If $f \in L^{p}(0, T ; X)$, take a sequence $\left\{h_{n}\right\}_{n \geq 1}$ such that $\left\|f-h_{n}\right\|_{L^{p}(0, T ; X)} \rightarrow 0$. By (49), the sequence $\left\{\left\langle\Gamma, h_{n}\right\rangle\right\}_{n \geq 1}$ is Cauchy and hence converges, so we set $\langle\Gamma, f\rangle:=\lim _{n \rightarrow \infty}\left\langle\Gamma, h_{n}\right\rangle$, this limit being independent of the sequence $h_{n}$. Using (48), it follows that $\Gamma$ is linear, and from (49) it is bounded,

$$
|\langle\Gamma, f\rangle| \leq\|v\|_{L^{q}(0, T)} \lim _{n \rightarrow \infty}\left\|h_{n}\right\|_{L^{p}(0, T ; X)}=\|v\|_{L^{q}(0, T)}\|f\|_{L^{p}(0, T ; X)},
$$

so that $\Gamma \in L^{p}(0, T ; X)^{*}$. By Theorem $\left[3.3\right.$, there exists $[\Phi] \in L_{w *}^{q}\left(0, T ; X^{*}\right)$ such that

$$
\langle\Gamma, f\rangle=\int_{0}^{T}\langle\Phi(s), f(s)\rangle d s \quad \text { for all } \quad f \in L^{p}(0, T ; X) .
$$

Define $\bar{\Psi}:[0, T] \rightarrow X^{*}$ by

$$
\bar{\Psi}(t)=\psi_{0}+\star \int_{0}^{t} \Phi(s) d s, \quad \text { so that } \quad[\bar{\Psi}] \in W_{w *}^{1, q}\left(0, T ; X^{*}\right) .
$$

Then, recalling (45) and (47), we have for each $x \in X$

$$
\begin{aligned}
\langle\bar{\Psi}(t), x\rangle & =\left\langle\psi_{0}, x\right\rangle+\int_{0}^{T}\left\langle\Phi(s), x \mathcal{X}_{[0, t]}(s)\right\rangle d s=\left\langle\psi_{0}, x\right\rangle+\left\langle\Gamma, x \mathcal{X}_{[0, t]}\right\rangle \\
& =\left\langle\psi_{0}, x\right\rangle+\int_{0}^{t} v_{x}(s) d s=\langle\Psi(t), x\rangle, \quad \text { a.e. } t \in[0, T],
\end{aligned}
$$

so that $\Psi \in[\bar{\Psi}]$ and thus $\Psi \in W_{w *}^{1, q}\left(0, T ; X^{*}\right)$, completing the proof for $q>1$.

Now set $q=1$ and suppose that $\left\{x_{n}\right\}_{n \geq 1}$ is dense in $X$. Then the set

$$
E=\bigcup_{n \geq 1}\left\{t \in(0, T): \lim _{h \rightarrow 0} \frac{1}{h} \int_{t}^{t+h} v_{x_{n}}(s) d s=v_{x_{n}}(t), \quad\left|v_{x_{n}}(t)\right| \leq v(t)\left\|x_{n}\right\|\right\}
$$

has full measure. For $t_{0} \in E$, define $\Phi\left(t_{0}\right)$ by $\left\langle\Phi\left(t_{0}\right), x_{n}\right\rangle=v_{x_{n}}\left(t_{0}\right)$ for each $n$. Then, as above, we check that $\Phi\left(t_{0}\right)$ is a bounded linear functional on the linear span of the set $\left\{x_{n}\right\}_{n \geq 1}$, with norm bounded by $v\left(t_{0}\right)$. This can be extended by continuity to a linear functional on all of $X$, with norm $\left\|\Phi\left(t_{0}\right)\right\|_{X^{*}} \leq v\left(t_{0}\right)$. Setting $\Phi\left(t_{0}\right)=0$ for $t_{0} \notin E$, we obtain a mapping $\Phi:[0, T] \rightarrow X^{*}$ such that $\left\langle\Phi(t), x_{n}\right\rangle=v_{x_{n}}(t)$ a.e. $t \in[0, T]$ for each $n$, and $\lim _{n \rightarrow \infty}\left\langle\Phi(t), x_{n}\right\rangle=$ $\langle\Phi(t), x\rangle$ for each $t$. This implies that $\Phi$ is weak* measurable, and $\|\Phi(t)\| \leq$ $v(t)$ implies that $[\Phi] \in L_{w *}^{1}\left(0, T ; X^{*}\right)$. Again define $\bar{\Psi}:[0, T] \rightarrow X^{*}$ by $\bar{\Psi}=$ $\psi_{0}+\star \int_{0}^{t} \Phi(s) d s$, so that $[\bar{\Psi}] \in W^{1,1}\left(0, T ; X^{*}\right)$.

For fixed $x \in X$, the set

$$
A_{x}=\left\{t:\langle\Psi(t), z\rangle=\left\langle\psi_{0}, z\right\rangle+\int_{0}^{t} v_{z}(s) d s, \quad \text { for } \quad z=x \text { and each } x_{n}\right\}
$$


has full measure. For $t \in A_{x}$ and any $n$, we have $\left\langle\bar{\Psi}(t), x_{n}\right\rangle=\left\langle\Psi(t), x_{n}\right\rangle$, so that

$$
\begin{aligned}
|\langle\bar{\Psi}(t)-\Psi(t), x\rangle| & =\left|\left\langle\bar{\Psi}(t), x-x_{n}\right\rangle-\left\langle\Psi(t), x-x_{n}\right\rangle\right| \\
& \leq\|\bar{\Psi}(t)\|\left\|x-x_{n}\right\|+\|\Psi(t)\|\left\|x-x_{n}\right\|,
\end{aligned}
$$

so that $\langle\bar{\Psi}(t), x\rangle=\langle\Psi(t), x\rangle$ for all $t \in A_{x}$. Since $x \in X$ is arbitrary, $\Psi$ and $\bar{\Psi}$ are weak*-equivalent and so $\Psi \in[\bar{\Psi}] \in W_{w *}^{1,1}\left(0, T ; X^{*}\right)$.

A.1. Relation to Brezis' Space. Given a Banach space $X$ and $1 \leq p \leq \infty$, Brezis defined the space $\widetilde{W}^{1, p}(0, T ; X)$ to be the set of all absolutely continuous functions $\Psi:[0, T] \rightarrow X$ for which the total variation function $t \rightarrow \mathbb{V}_{\Psi}(t)$ is absolutely continuous on $[0, T]$, and such that the scalar pointwise derivative $\frac{d \mathbb{V}_{\Psi}}{d t} \in L^{p}(0, T)$. In particular, for $\Psi \in \widetilde{W}^{1, p}(0, T ; X)$, for all $0 \leq s<t \leq T$, we have

$$
\|\Psi(t)-\Psi(s)\| \leq \mathbb{V}_{\Psi}(t)-\mathbb{V}_{\Psi}(s)=\int_{s}^{t} \frac{d}{d t} \mathbb{V}_{\Psi}(\tau) d \tau .
$$

Brezis proved that $W^{1, p}(0, T ; X) \subset \widetilde{W}^{1, p}(0, T ; X)$, and if $X$ is reflexive, the two spaces coincide, $W^{1, p}(0, T ; X)=\widetilde{W}^{1, p}(0, T ; X) \underline{\mathbf{3}}$.

We have shown in Theorems 3.6 and 3.4 that each $\Psi \in W_{w *}^{1, q}\left(0, T ; X^{*}\right)$ has an absolutely continuous representative $\bar{\Psi}$, and that this in turn satisfies $\bar{\Psi} \in \widetilde{W}^{1, q}\left(0, T ; X^{*}\right)$. We now show that the converse is also true, that is, if $\Psi \in \widetilde{W}^{1, q}\left(0, T ; X^{*}\right)$, then $[\Psi] \in W_{w *}^{1, q}\left(0, T ; X^{*}\right)$.

Theorem A.2. For $1<q \leq \infty$, the canonical mapping $\widetilde{W}^{1, q}\left(0, T ; X^{*}\right) \rightarrow$ $W_{w *}^{1, q}(0, T ; X)$, given by $\Psi \mapsto[\Psi]$, is injective, onto and norm-preserving. The same conclusion holds for $q=1$ provided $X$ is separable.

Proof. Suppose that $\Psi \in \widetilde{W}^{1, q}\left(0, T ; X^{*}\right)$ and fix $x \in X$. Then the numerical function

$$
z_{x}(t):=\langle\Psi(t), x\rangle:[0, T] \rightarrow \mathbb{R}
$$

is absolutely continuous, so its pointwise derivative $\frac{d z_{x}}{d x}$ is defined $\lambda$-a.e., belongs to $L^{1}(0, T)$, and

$$
\langle\Psi(t), x\rangle=\langle\Psi(s), x\rangle+\int_{s}^{t} \frac{d z_{x}}{d t}(\tau) d \tau \quad \text { for all } \quad 0 \leq s<t \leq T .
$$

Now, using (50), we have for all $0 \leq s<t \leq T$,

$$
\left|\int_{s}^{t} \frac{d z_{x}}{d t}(\tau) d \tau\right|=|\langle\Psi(t)-\Psi(s), x\rangle| \leq\|x\| \int_{s}^{t} \frac{d}{d t} \mathbb{V}_{\Psi}(\tau) d \tau,
$$

which in turn implies

$$
\left|\frac{d z_{x}}{d t}(t)\right| \leq\|x\| \frac{d}{d t} \mathbb{V}_{\Psi}(t) \quad \text { a.e. } t \in[0, T] .
$$

Recalling that the variation satisfies $\frac{d}{d t} \mathbb{V}_{\Psi}(\cdot) \in L^{q}(0, T)$, the result now follows from Theorem A.1 


\section{References}

1. S. Bianchini and A. Bressan, Vanishing viscosity solutions of nonlinear hyperbolic systems, Ann. Math. 161 (2005), 223-342.

2. A. Bressan, Hyperbolic systems of conservation laws: The one-dimensional Cauchy problem, Oxford University Press, 2000.

3. H. Brezis, Oprateurs maximaux monotones et semi-groupes de contractions dans les espaces de hilbert, North-Holland, 1973.

4. P. Cembranos and J. Mendoza, Banach spaces of vector-valued functions, SpringerVerlag, 1997.

5. Constantine M. Dafermos, Hyperbolic conservation laws in continuum physics, Springer, 2000 .

6. V. Danilov and V. Shelkovich, Delta-shock wave type solution of hyperbolic systems of conservation laws, Quart. Appl. Math. 63 (2005), 401-427.

7. J. Diestel and J.J. Uhl, Vector measure, AMS, 1989.

8. J. Giesselmann and A.E. Tzavaras, Singular limiting induced from continuum solutions and the problem of dynamic cavitation, Arch. Rational Mech. Anal. 212 (2014), 241-281.

9. J. Glimm, Solutions in the large for nonlinear hyperbolic systems of equations, Comm. Pure Appl. Math. 18 (1965), 697-715.

10. B. Keyfitz and H. Kranzer, A viscosity approximation to a system of conservation laws with no classical Riemann solution, Lecture Notes in Mathematics 1402 (1989), 185197.

11. P.D. Lax, Hyperbolic systems of conservation laws, II, Comm. Pure Appl. Math. 10 (1957), 537-566.

12. C. De Lellis and L. Szekelyhidi, On admissibility criteria for weak solutions of the Euler equations, Arch. Rational Mech. Anal. 195 (2010), 225-260.

13. A. Majda, Compressible fluid flow and systems of conservation laws in several space variables, Applied Mathematical Sciences, no. 53, Springer-Verlag, 1984.

14. A. Miroshnikov and R. Young, Weak* solutions II: The vacuum in Lagrangian gas dynamics, to appear, SIAM J. Math. Anal., 2017.

15. A. Miroshnikov and R. Young, Weak* solutions III: A convergent front tracking scheme, In preparation, 2016.

16. A. Miroshnikov and R. Young, Weak* solutions IV: A general unified approach for balance laws, In preparation, 2016.

17. J. Rauch, $B V$ estimates fail for most quasilinear hyperbolic systems in dimensions greater than one, Commun. Math. Phys. 106 (1986), 481-484.

18. H. L. Royden and P.M. Fitzpatrick, Real analysis, 4th ed., Prentice Hall, 2010.

19. V. Scheffer, An inviscid flow with compact support in space-time, J. Geom. Anal. 3(4) (1993), 343-401.

20. Michael Sever, Distribution solutions of nonlinear systems of conservation laws, Memoirs Amer. Math. Soc. 889 (2007).

21. A. Shnirelman, On the nonuniqueness of weak solution of the Euler equation, Comm. Pure. Appl. Math L (1997), 1261-1286.

22. A. Ionescu Tulcea and C. Ionescu Tulcea, Topics in the theory of lifting, Springer, 1969.

23. Robin Young, Sup-norm stability for Glimm's scheme, Comm. Pure Appl. Math. 46 (1993), 903-948.

24. Robin Young, The p-system II: The vacuum, Evolution Equations (Warsaw) (R. Picard, M. Reissig, and W. Zajaczkowski, eds.), Banach Center, 2001, pp. 237-252.

25. Robin Young, Composite wave interactions and the collapse of vacuums in gas dynamics, J. Diff. Eq. 252 (2012), 5129-5154. 
Department of Biostatistics and Epidemiology, Univ of Massachusetts Amherst CURRENT address: Department of Mathematics, UCLA Department of Mathematics and Statistics, Univ of Massachusetts Amherst

E-mail address: amiroshn@gmail.com, young@math.umass.edu 